\title{
The VIMOS VLT deep survey ${ }^{\star}$
}

\section{First epoch VVDS-deep survey: 11564 spectra with $17.5 \leq I_{A B} \leq 24$, and the redshift distribution over $0 \leq z \leq 5$}

\author{
O. Le Fèvre ${ }^{1}$, G. Vettolani ${ }^{2}$, B. Garilli ${ }^{3}$, L. Tresse ${ }^{1}$, D. Bottini ${ }^{3}$, V. Le Brun ${ }^{1}$, D. Maccagni ${ }^{3}$, J. P. Picat ${ }^{4}$,
}

R. Scaramella ${ }^{2}$, M. Scodeggio ${ }^{3}$, A. Zanichelli ${ }^{2}$, C. Adami ${ }^{1}$, M. Arnaboldi ${ }^{5}$, S. Arnouts ${ }^{1}$, S. Bardelli ${ }^{8}$, M. Bolzonella ${ }^{9}$, A. Cappi ${ }^{8}$, S. Charlott ${ }^{6,11}$, P. Ciliegi ${ }^{8}$, T. Contini $^{4}$, S. Foucaud ${ }^{3}$, P. Franzetti ${ }^{3}$, I. Gavignaud ${ }^{4,12}$, L. Guzzo ${ }^{10}$, O. Ilbert ${ }^{1,8}$, A. Iovino ${ }^{10}$, H. J. McCracken ${ }^{6}$, B. Marano ${ }^{9}$, C. Marinoni ${ }^{1,10}$, G. Mathez ${ }^{4}$, A. Mazure ${ }^{1}$, B. Meneux ${ }^{1}$, R. Merighi ${ }^{8}$, S. Paltani ${ }^{1}$, R. Pello ${ }^{4}$, A. Pollo ${ }^{10}$, L. Pozzetti ${ }^{8}$, M. Radovich ${ }^{5}$, G. Zamorani ${ }^{8}$, E. Zucca ${ }^{8}$, M. Bondi ${ }^{2}$, A. Bongiorno ${ }^{9}$, G. Busarello ${ }^{5}$, F. Lamareille ${ }^{4}$, Y. Mellier $^{6}$, P. Merluzzi ${ }^{5}$, V. Ripepi ${ }^{5}$, and D. Rizzo ${ }^{4}$

${ }^{1}$ Laboratoire d'Astrophysique de Marseille, OAMP, Université de Provence, UMR 6110, Traverse du Siphon-Les trois Lucs, 13012 Marseille, France, e-mail: olivier. lefevre@oamp. fr

2 Istituto di Radio-Astronomia - INAF, 40129 Bologna, Italy

3 IASF - INAF, 20133 Milano, Italy

${ }^{4}$ Laboratoire d'Astrophysique - Observatoire Midi-Pyrénées, 31400 Toulouse, France

5 Osservatorio Astronomico di Capodimonte - INAF, via Moiariello 16, 80131 Napoli, Italy

${ }^{6}$ Institut d'Astrophysique de Paris, UMR 7095, 98bis Bd. Arago, 75014 Paris, France

7 Observatoire de Paris, LERMA, UMR 8112, 61 Av. de l'Observatoire, 75014 Paris, France

8 Osservatorio Astronomico di Bologna - INAF, via Ranzani 1, 40127 Bologna, Italy

9 Università di Bologna, Dipartimento di Astronomia, via Ranzani, 1, 40127 Bologna, Italy

10 Osservatorio Astronomico di Brera - INAF, via Brera 28, 20121 Milan, Italy

11 Max-Planck-Institut für Astrophysik, 85741 Garching, Germany

12 European Southern Observatory, Karl-Schwarzschild-Strasse 2, 85748 Garching bei München, Germany

Received 6 September 2004 / Accepted 22 February 2005

\begin{abstract}
This paper presents the "First Epoch" sample from the VIMOS VLT Deep Survey (VVDS). The VVDS goals, observations, data reduction with the VIPGI pipeline and redshift measurement scheme with KBRED are discussed. Data have been obtained with the VIsible Multi Object Spectrograph (VIMOS) on the ESO-VLT UT3, allowing us to observe $\simeq 600$ slits simultaneously at a spectral resolution $R \simeq 230$. A total of 11564 objects have been observed in the VVDS-02h and VVDSCDFS "Deep" fields over a total area of $0.61 \mathrm{deg}^{2}$, selected solely on the basis of apparent magnitude $17.5 \leq I_{A B} \leq 24$. The VVDS efficiently covers the redshift range $0<z \leq 5$. It is successfully going through the "redshift desert" $1.5<z<2.2$, while the range $2.2<z<2.7$ remains of difficult access because of the VVDS wavelength coverage. A total of 9677 galaxies have a redshift measurement, 836 objects are stars, 90 objects are AGN, and a redshift could not be measured for 961 objects. There are 1065 galaxies with a measured redshift $z \geq 1$.4. When considering only the primary spectroscopic targets, the survey reaches a redshift measurement completeness of $78 \%$ overall (93\% including less reliable flag 1 objects), with a spatial sampling of the population of galaxies of $\sim 25 \%$ and $\sim 30 \%$ in the VVDS- $02 \mathrm{~h}$ and VVDS-CDFS respectively. The redshift accuracy measured from repeated observations with VIMOS and comparison to other surveys is $\sim 276 \mathrm{~km} \mathrm{~s}^{-1}$.

From this sample we are able to present for the first time the redshift distribution of a magnitude-limited spectroscopic sample down to $I_{A B}=24$. The redshift distribution $N(z)$ has a median of $z=0.62, z=0.65, z=0.70$, and $z=0.76$, for magnitudelimited samples with $I_{A B} \leq 22.5,23.0,23.5$ and 24.0 respectively. A high redshift tail above redshift 2 and up to redshift 5 becomes readily apparent for $I_{A B}>23.5$, probing the bright star-forming population of galaxies. This sample provides an unprecedented dataset to study galaxy evolution over $\sim 90 \%$ of the life of the universe.
\end{abstract}

Key words. cosmology: observations - galaxies: high-redshift - galaxies: distances and redshift - surveys - galaxies: evolution - cosmology: large scale structure of Universe

\section{Introduction}

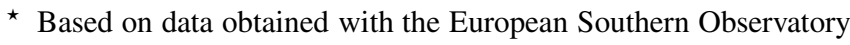
Very Large Telescope, Paranal, Chile, program 070.A-9007(A), and on data obtained at the Canada-France-Hawaii Telescope, operated by the CNRS of France, CNRC in Canada and the University of Hawaii.
Understanding how galaxies and large scale structures formed and evolved is one of the major goals of modern cosmology. In order to identify the relative contributions of the various physical processes at play and the associated timescales, 
a comprehensive picture of the evolutionary properties of galaxies and AGN, and their distribution in space, is needed over a large volume and a large cosmic time base.

Samples of high redshift galaxies known today reach several hundred to a few thousand objects in the redshift range $0.5-4$, and statistical analysis suffers from small number statistics, small explored volumes and different selection biases for each sample, which prevent detailed analysis. In contrast, in the local universe, large surveys like the 2dFGRS (Colless et al. 2001) and the Sloan SDSS (Abazajian et al. 2003) contain from 250000 up to one million galaxies and reach a high level of accuracy in measuring the fundamental parameters of the galaxy and AGN populations. In a similar way, we need to gather large numbers of galaxies at high redshifts to accurately quantify the normal galaxy population through the measurement of e.g. the luminosity function, correlation function and star formation rate, for different galaxy types and in environments ranging from low density to dense cluster cores.

We are currently conducting the VIRMOS VLT Deep Survey (VVDS), a coherent approach to study the evolution of galaxies, large scale structures and AGN. The observational goals are: (1) a "wide" survey: 100000 galaxies and AGN observed at low spectral resolution $R \simeq 230$, in $16 \mathrm{deg}^{2}$, to a limiting magnitude $I_{A B}=22.5$ and reaching redshifts up to $\sim 1.3$; (2) a "deep" survey: 235000 galaxies and AGN observed at $R \simeq 230$ in $2 \mathrm{deg}^{2}$ and brighter than $I_{A B}=24$, to map evolution over $0 \leq z \leq 5$, or $90 \%$ of the age of the universe; (3) an "ultradeep" survey: 1000 galaxies and AGN brighter than $I_{A B}=25$, this will probe deep into the luminosity function (3 mag below $M^{*}$ at $z=1$ ). In addition, we intend to conduct a selected high redshift cluster survey following up the clusters identified in the "wide" and "deep" surveys, and a high spectral resolution survey, $R \sim 2500-5000$, on a sub-sample of 10000 galaxies selected from the "wide" and "deep" surveys. The observing strategy that we have devised allows us to carry out these goals in an optimized and efficient approach.

Guaranteed Time Observations allow completion of $~ 40 \%$ of the original survey goals. A Large Program is being proposed to the European Southern Observatory to complete the VVDS as originally planned.

We describe here the survey strategy and the status of the observations in the first epoch "Deep" survey. We detail the scientific motivation in Sect. 2, the survey strategy in Sect. 3, the "first epoch" observations in Sect. 4, the pipeline processing of the VIMOS data with VIPGI in Sect. 5, the methods followed to measure redshifts in Sect. 6 and the "first epoch" VVDSDeep sample in Sect. 7. After a description of the galaxy population probed by the VVDS-Deep in Sect. 8, we present the redshift distribution of magnitude-limited samples as deep as $I_{A B}=24$ in Sect. 9, and we conclude in Sect. 10.

We have used a Concordance Cosmology with $h=0.7$, $\Omega_{\mathrm{m}}=0.3$, and $\Omega_{\Lambda}=0.7$ throughout this paper.

\section{Survey goals}

\subsection{Science background}

The current theoretical picture of galaxy formation and evolution and of large scale structure growth in the universe is well advanced. In contrast, while existing observations already provide exciting views on the properties and distribution of high redshift objects (Lilly et al. 1995a; Le Fèvre et al. 1995; Steidel et al. 1996; $\quad$ Steidel et al. 1998; Lilly et al. 1996; Madau et al. 1998; Cimatti et al. 2002; Abraham et al. 2004; Wolf et al. 2003) the samples remain too small, incomplete, affected by large and sometimes degenerate errors from photometric redshifts measurements, or targeted toward specific populations, making it hazardous to relate the evolution observed in different populations at different epochs, study how evolution depends upon luminosity, type or local environment of the populations, and compare results to theoretical predictions.

The massive efforts conducted by the $2 \mathrm{dF}$ and Sloan surveys (see e.g. Colless et al. 2001, http://mso . anu.edu . au/ 2dFGRS/; and Abazajian et al. 2003, http://www.sdss . org/) have been aimed at mapping the distribution and establish the properties of more than one million galaxies in our local environment up to redshifts $\sim 0.3$. As we get to know better our local universe, deep surveys on volumes comparable to the 2dFGRS or SDSS at look back times spanning a large fraction of the age of the universe are required to give access to the critical time dimension and trace back the history of structure formation on scales from galaxies up to filaments with $\sim 100 h^{-1}$ Mpc.

This reasoning forms the basis of the VIRMOS-VLT Deep Survey (VVDS). It is intended to probe the universe at increasingly higher redshifts to establish the evolutionary sequence of galaxies, AGN, clusters and large scale structure, and provide a statistically robust dataset to challenge current and future models, from one single dataset. Unlike all previous surveys at the proposed depth, the VVDS is based on a simple and easy to model selection function: the sample is selected only on the basis of $I$ band magnitude, as faint as $I_{A B}=24$ to cover the largest possible redshift range. Although this produces an obvious bias by selecting increasingly brighter galaxies when going to higher redshifts, this selection criterion allows us to perform a complete census of the galaxy population in a given volume of the universe, above a fixed and well-defined luminosity.

\subsection{Science motivation}

The VVDS is designed to address the following goals:

(1) Formation and evolution of galaxies: the goals are to study the evolution of the main population of galaxies in the redshift range $0<z<5+$ from a complete census of the population of galaxies. Several indicators of the evolution status of the galaxy population will be computed as a function of redshift and spectral type: counts and colors, $N(z$, type), luminosity and mass function (including vs. spectral type, local galaxy density), star formation rate from various indicators, spectrophotometric properties, merger rate, etc. These indicators will be 
combined to establish the evolutionary properties of galaxies with unprecedented statistical accuracy. The multi-wavelength approach (radio at VLA, X-ray with XMM, far-IR with Spitzer, and UV with Galex) on the 0226-04 and CDFS deep fields will allow us to probe galaxy evolution from the signatures of different physical processes. HST imaging is on-going on the extended VVDS-10h field selected for the HST-COSMOS program.

From the main low resolution survey, it will be possible to select a well defined sub-sample of high redshift galaxies to be observed at high spectral resolution to determine the fundamental plane of elliptical galaxies at redshifts greater than 1 , and up to $z \sim 2$ for star forming galaxies.

(2) Formation and evolution of large scale structures: the formation and evolution of large-scale structures (LSS) is yet to be explored on scales $50-100 \mathrm{Mpc}$ at redshifts $0.5<$ $z<5+$. The goal is here to map the cosmic web structure back to unprecedented epochs. From this, a variety of statistical indicators of galaxy clustering and dynamics can be computed, including 3D galaxy density maps, the 2-point correlation functions $\xi(r)$ and $\xi\left(r_{\mathrm{p}}, \pi\right)$ and their projections $w_{\mathrm{p}}\left(r_{\mathrm{p}}\right)$, power spectrum, counts in cells and other higher order statistics. The direct comparison of the observed probability distribution function (PDF) of galaxies with the PDF of dark matter halos predicted in the framework of cosmological models will be used to yield a measurement of the evolution of galaxy biasing. The dependence of clustering evolution on galaxy type and luminosity will be investigated. The data will enable comparison of the 3D galaxy distribution with the mass maps produced by weak-shear. These indicators will be combined to form a coherent picture of LSS evolution from $z \sim 4-5$, which will be compared to current large $\mathrm{N}$-body simulations/semi-analytical model predictions.

(3) Formation and evolution of AGN: while the evolution of the bright AGNs is well constrained by the large SDSS and $2 \mathrm{dF}$ AGN surveys out to large redshifts (Boyle et al. 2000), constraints on fainter AGN are weak. With no a priori selection of the survey targets based e.g. on image compactness or color, the detection of AGNs along with the main population of galaxies is feasible (Schade et al. 1995). This will allow us to study the evolution of the AGN population, in particular the evolution of the luminosity function of QSOs out to $z \sim 5$, and the evolution of the AGN fraction in the galaxy population with redshift. We will also establish the clustering properties of AGN. The 0226-04 and CDFS deep fields are of particular interest because of the multi-wavelength approach including X-ray, radio and far-IR observations.

(4) Formation and evolution of clusters of galaxies: the known sample of high redshift clusters of galaxies above $z=0.5$ is still small, and volume-limited samples are difficult to obtain. In the VVDS samples, we expect to identify several dozen clusters (depending on cosmological models), half of them at a redshift above 0.5 . We will acquire both large-scale velocity information from MOS spectra, and detailed core mapping from IFU spectroscopy. This will enable us to describe both the dynamical state of the clusters and the spectrophotometric evolution of the galaxy population in clusters. The combination of these data with XMM data and weak shear analysis will be particularly powerful to establish the mass properties. We will aim to derive the evolution of the cluster space density.

These scientific goals have been used to establish the observational strategy described below.

\section{Survey strategy}

\subsection{VVDS surveys}

The science goals require a large number of objects over large, deep volumes. They are all addressed in a consistent way by the following observational strategy: (1) wide survey: 100000 galaxies and AGN observed in the 4 survey fields to $I_{A B}=22.5, R \simeq 230$, the survey will sample $\sim 20 \%$ of the galaxies to this magnitude; exposure times are 3000 s. (2) Deep survey: 35000 galaxies and AGN observed in the 0226-04 field and CDFS to $I_{A B}=24, R \simeq 230$, the survey will sample $25 \%$ of the galaxies to this magnitude; exposure times are $16200 \mathrm{~s}$; (3) 1000 galaxies and AGN observed in the 0226-04 field to $I_{A B}=25, R \sim 200$; (4) 10000 galaxies in all fields to $I_{A B}=22.5, R=2500-5000$; (5) 50-100 cluster cores in all fields to $I_{A B}=24, R=1000$.

Efficient observations can be carried out with $\sim 500$ 600 galaxies observed in one single spectroscopic observation for the "deep" survey and about 350-400 galaxies in the "wide" survey. The sample is designed to bring down the statistical noise, to measure e.g. the luminosity function $\phi *, M^{*}$, or $\alpha$ parameters, or correlation length $r_{0}(z)$ to better than $10 \%$ at any of 7 time steps.

\subsection{Multi-wavelength surveys}

In addition to the $U, B V R I, J K$ surveys conducted by the VVDS team (Le Fèvre et al. 2004a; McCracken et al. 2003; Radovich et al. 2003; Iovino et al. 2005, respectively), multiwavelength surveys are carried out by the VVDS team or under data exchange agreements with other teams. Some of the VVDS fields have been observed at $1.4 \mathrm{GHz}$ at the VLA (Bondi et al. 2003), with XMM (Pierre et al. 2003), by Galex (Arnouts et al. 2004; Schiminovich et al. 2004) and are being observed by Spitzer (Lonsdale et al. 2003).

\subsection{The VIMOS instrument}

The VIsible Multi-Object Spectrograph is installed on the European Southern Observatory (ESO) Very Large Telescope (VLT), at the Nasmyth focus of the VLT unit telescope 3 "Melipal" (Le Fèvre et al. 2003a). VIMOS is a 4-channel imaging spectrograph, each channel (a "quadrant") covering $\sim 7 \times 8 \operatorname{arcmin}^{2}$ for a total field of view (a "pointing") of $\sim 218 \operatorname{arcmin}^{2}$. Each channel is a complete spectrograph with the possibility to insert slit masks $\sim 30 \times 30 \mathrm{~cm}^{2}$ each at the entrance focal plane, broad band filters or grisms to produce spectra on a $2048 \times 4096$ pixels $^{2}$ EEV CCD.

The pixel scale is 0.205 arcsec/pixel, providing excellent sampling of the Paranal mean image quality and Nyquist sampling for a slit 0.5 arcsec in width. The spectral resolution ranges from $\sim 200$ to $\sim 5000$. Because the instrument field at 
Table 1. VVDS fields: positions and available optical and near-infrared photometry.

\begin{tabular}{|c|c|c|c|c|c|c|}
\hline Field & $\alpha_{2000}$ & $\delta_{2000}$ & $b$ & $l$ & Survey mode & Multi-wavelength data \\
\hline $\begin{array}{l}0226-04 \\
\text { VVDS - 02h }\end{array}$ & $02 \mathrm{~h} 26 \mathrm{~m} 00.0 \mathrm{~s}$ & $-04 \operatorname{deg} 30^{\prime} 00^{\prime \prime}$ & -58.0 & -172.0 & Deep & $\begin{array}{c}B, V, R, I^{a} \\
J, K^{b} \\
U^{c}\end{array}$ \\
\hline $1003+01$ & $10 \mathrm{~h} 03 \mathrm{~m} 00.0 \mathrm{~s}$ & $+01 \operatorname{deg} 30^{\prime} 00^{\prime \prime}$ & 42.6 & 237.8 & Wide & $\begin{array}{l}B, V, R, I^{a} \\
\quad J, K^{b}\end{array}$ \\
\hline $1400+05$ & $14 \mathrm{~h} 00 \mathrm{~m} 00.0 \mathrm{~s}$ & $+05 \operatorname{deg} 00^{\prime} 00^{\prime \prime}$ & 62.5 & 342.4 & Wide & $\begin{array}{c}B, V, R, I^{a} \\
\quad J, K^{b}\end{array}$ \\
\hline $2217+00$ & $22 \mathrm{~h} 17 \mathrm{~m} 50.4 \mathrm{~s}$ & $+00 \operatorname{deg} 24^{\prime} 00^{\prime \prime}$ & -44.0 & 63.3 & Wide & $\begin{array}{l}B, V, R, I^{a} \\
\quad J, K^{b}\end{array}$ \\
\hline $\begin{array}{l}\text { CDFS } \\
\text { VVDS - CDFS }\end{array}$ & 03h32m28.0s & $-27 \operatorname{deg} 48^{\prime} 30^{\prime \prime}$ & -54.5 & 223.5 & Deep & $\begin{array}{c}B, V, R, I^{d} \\
H S T B, V, R, I^{e}\end{array}$ \\
\hline
\end{tabular}

${ }^{a}$ (Le Fèvre et al. 2004a), ${ }^{b}$ (Iovino et al. 2005), ${ }^{c}$ (Radovich et al. 2003), ${ }^{d}$ (Arnouts et al. 2001), ${ }^{e}$ (Giavalisco et al. 2004).

the Nasmyth focus of the VLT is large $(\simeq 1 \mathrm{~m})$, there is no atmospheric dispersion compensator. This requires us to limit observations to airmasses below 1.7.

In the MOS mode of observations, short "pre-images" are taken ahead of the observing run. These are cross-correlated with the user catalog to match the instrument coordinate system to the astrometric reference of the user catalog. Slit masks are prepared using the VMMPS tool, with an automated optimization of slit numbers and position (see Bottini et al. 2005).

\subsection{VVDS fields}

The 4 main fields of the VVDS have been selected at high galactic latitude, and are spread around the celestial equator to allow year round survey observations. Each field is $2 \times 2 \mathrm{deg}^{2}$. The imaging is deep enough to select the VIRMOS targets at the survey depth without selection effects (see Sect. 3.6). We have included the Chandra Deep Field South (CDFS, Giacconi et al. 2002), which is the target of Chandra, HST, XMM and Spitzer multi-wavelength observations, to complement the work of the GOODS consortium (Giavalisco et al. 2004).

The fields positions and available optical and near-infrared imaging data are summarized in Table 1.

\subsection{Field coverage: VIMOS pointing layout for the VVDS-Deep}

The VVDS "Deep" survey is conducted in the central $1.2 \mathrm{deg}^{2}$ area of the VVDS-02h field and in the CDFS. In the VVDS-02h area, we have devised a scheme which allows uniform coverage of a central area of $0.92 \times 1.33 \mathrm{deg}^{2}$, with 4 passes of the instrument, i.e. each point on the sky has 4 chances of being selected for spectroscopy, using 66 pointings spaced by (2 arcmin, 2 arcmin $)$ in $(\alpha, \delta)$ as shown in Fig. 1.

The VIMOS multiplex allows measurement of on average $\sim 540$ spectra per pointing at the magnitude $I_{A B} \leq 24$;

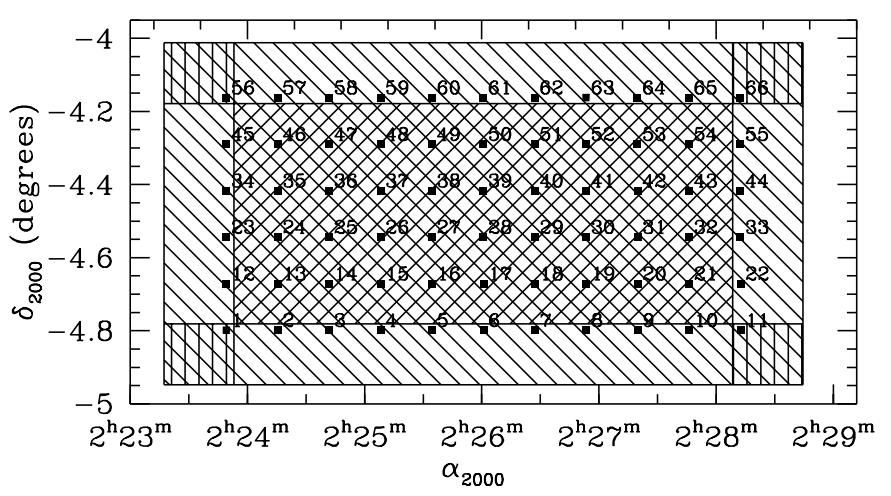

Fig. 1. The planned layout and identification numbers of the VIMOS pointings for the VVDS "Deep" survey in the VVDS-02h. A total of 66 pointings is planned, placed on a grid with $(2,2)$ arcmin spacing in $(\alpha, \delta)$. This layout allows observation of the central area (cross hatched) 4 times, and edge areas twice (hatched) except for the 4 corners which are observed once (triple hatched). See Table 2 for the list of already observed fields and Fig. 12 for the layout of objects already observed.

this pointing strategy therefore enables observation of 35000 spectra in the "deep" area.

The pointing layout of the VVDS-Wide survey includes 96 pointings to cover $2 \times 2 \mathrm{deg}^{2}$ with a grid of pointings overlapping twice; it will be presented elsewhere (Garilli et al., in preparation).

\subsection{I-band magnitude-limited sample}

The selection function used to identify targets to be observed spectroscopically has a deep impact on the final content of the survey. We have taken the classical approach of a pure magnitude selection of the sample, without any other color or shape criteria: the VVDS "Deep" survey is limited to $17.5 \leq I_{A B} \leq 24$ and the "Wide" survey is limited to $17.5 \leq$ $I_{A B} \leq 22.5$. The $I$ band selection is made as a compromise to select the galaxy population from the flux emitted by the "old" stellar content above $4000 \AA$ up to $z \sim 1$. At redshifts 
above $z=1$, this selection criterion implies that galaxies are selected from the continuum emission below $4000 \AA$, going increasingly towards the UV as redshift increases.

We do not to impose any shape criteria on the sample selection. This is dictated by the main requirement to keep QSOs, compact AGN, or compact galaxies in the sample. The "stargalaxy" separation from most photometry codes is known to be only valid for relatively bright magnitudes, with the locus of stars and galaxies in a "magnitude-shape" diagram overlapping for the faintest magnitudes. Eliminating star-like objects on the basis of the shape of the image profile alone would thus eliminate a significant part of the AGN and compact galaxy population with little control over the parent population or redshift domain which is lost. We can test this a posteriori from our unbiased spectroscopic sample; this will be presented elsewhere.

The magnitude selection sets strong requirements on the photometric catalog necessary as input to select the spectroscopic target list. We have conducted the CFH12k-VVDS imaging survey at CFHT to cover the 4 VVDS fields in $B, V, R$ and $I$ bands (Le Fèvre et al. 2004a). The depth of the imaging survey is $\sim 1$ mag deeper than the spectroscopic survey limit, which ensures that there is no a-priori selection against low surface brightness galaxies (McCracken et al. 2003). The multicolor information is used only after the spectroscopic observations, e.g. to help determine k-corrections (Ilbert et al. 2005). In addition to the core $B, V, R, I$ photometric data, $U$ band photometry (Radovich et al. 2003) and $J, K^{\prime}$ photometry (Iovino et al. 2005) have been obtained for part of the fields.

\section{First epoch observations: the VVDS-Deep survey in VVDS-0226-04}

\subsection{Preparation of VIMOS observations: VMMPS and database tools}

The preparation of VVDS observations is done from the selected list of pointings. The VVDS database implemented under Oracle is used to extract the list of targets allowed in a given pointing, ensure a secure follow-up of the work on each pointing and in particular to treat the overlapping observations. For each pointing a short $R$-band image is taken with VIMOS ahead of the spectroscopic observations. Upon loading in the database, this "pre-image" is automatically flat-fielded and the detection algorithm of Sextractor (Bertin \& Arnouts 1996) is run to identify the brightest $\sim 80$ sources, and a catalog of bright sources is produced with coordinates in the VIMOS CCD coordinate system.

The next steps are conducted with the VMMPS tool to design the slit mask layout (Bottini et al. 2005). The positions of the sources identified from the "pre-image" are cross-correlated with the deep VVDS source catalog (Le Fèvre et al. 2004a) to derive the transformation matrix from the $(\alpha, \delta)$ sky reference frame of the VVDS catalog to the $\left(X_{\mathrm{CCD}}, Y_{\mathrm{CCD}}\right)$ VIMOS instrumental coordinate system. After placing two reference apertures on bright stars for each pointing quadrant (see below), slits are then assigned to a maximum number of sources in the photometric catalog.

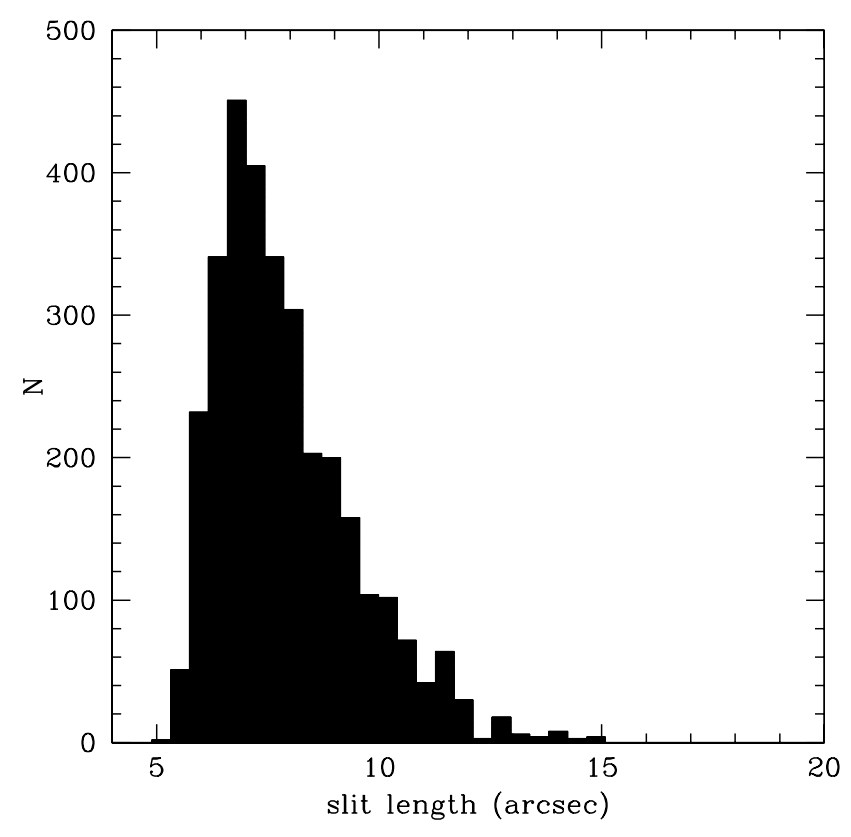

Fig. 2. Distribution of slit length in 6 of the VVDS-02h pointings.

The automated SPOC (Slit Positioning Optimisation Code, see Bottini et al. 2005) algorithm is run to optimize the number of slits given the geometrical and optical constraints of the VIMOS set-up (Bottini et al. 2005). We have designed masks with slits of one arcsecond width, and have forced that a minimum of 1.8 arcsec of sky is left on each side of a targeted object to allow for accurate sky background fitting and removal during later spectroscopic data processing. The typical distribution of slit length in the VVDS is shown in Fig. 2. On average, a VVDS-Deep pointing of 4 quadrant masks contains about 540 slits.

Upon completion of this step, Aperture Definition files in Pixels (ADP) are produced. ADPs contain the positions, length and width of all slits to be observed, produced using the VIMOS internal transformation matrix from the mask focal plane to the CCD focal plane to transform the coordinates of any VVDS source in the photometric catalog to VIMOS mask coordinates. The ADPs are then sent to the Mask Manufacturing Machine (MMU, Conti et al. 2001) for the masks to be cut and stored in the VIMOS "mask cabinets" ready for observation. Database handling of objects already targeted ensures no duplication of observations in overlapping areas of adjacent pointings.

\subsection{VIMOS observations}

We have built Observing Blocks (OBs) using the standard "Jitter" template, with 5 steps along the slit, each separated by 0.7 arcsec, at $-1.4,-0.7,0,0.7,1.4$ arcsec from the nominal on-target position. Moving the objects along the slit considerably improves the data processing in the presence of the strong fringing produced by the thin EEV CCDs used in VIMOS (see below).

We have been using the LRRED grism together with the RED cutoff filter, which limits the bandpass and order overlap. 

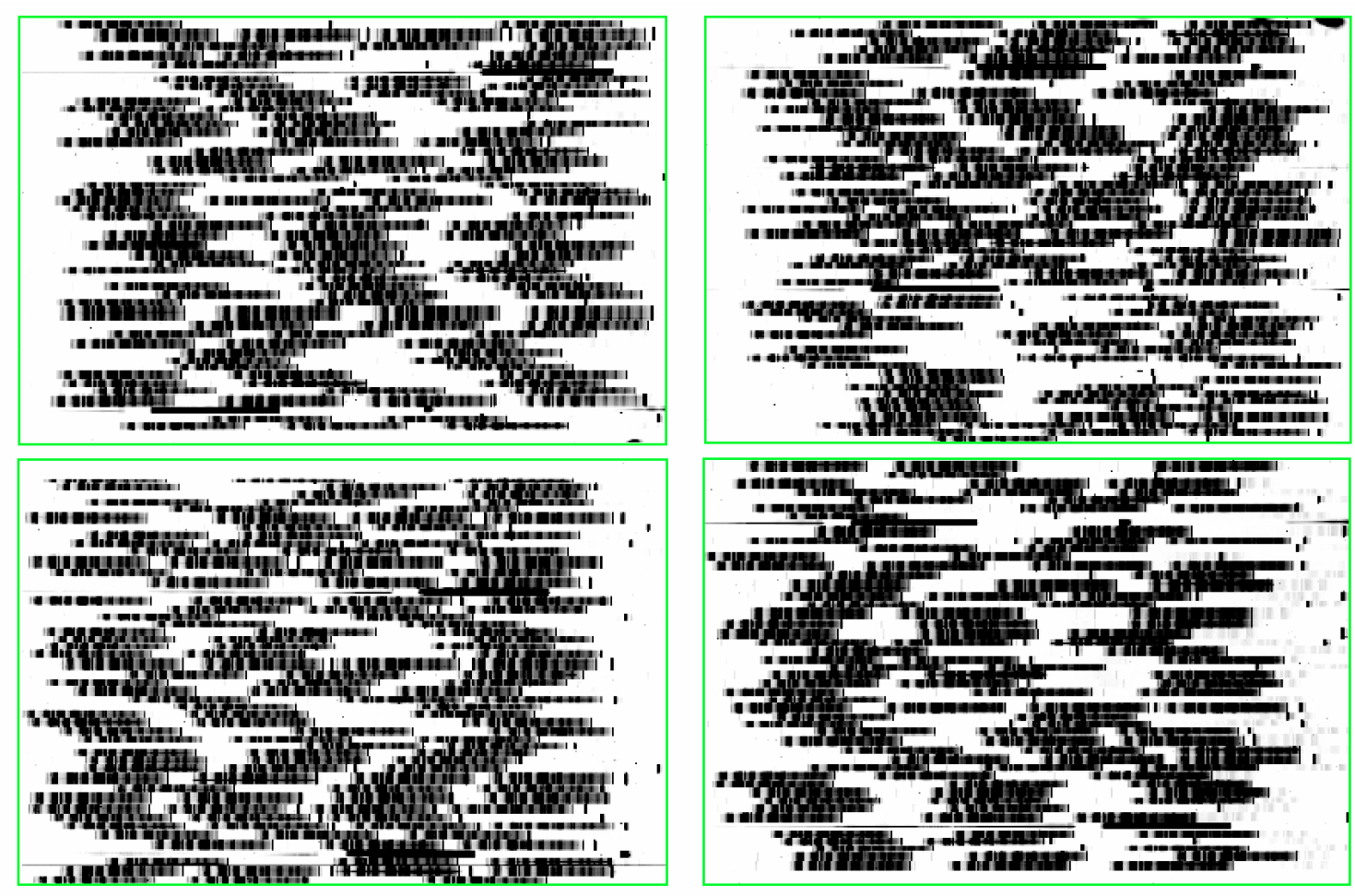

Fig. 3. Typical layout of spectra in the 4 quadrants of a VVDS-Wide pointing; more than 550 are observed on average.

The useful wavelength range is 5500 to $9400 \AA$. With one arcsecond slits, the resolution measured at $7500 \AA$ is $33 \AA$, or $R=227$, and the dispersion is $7.14 \AA$ /pixel.

In the "Deep" survey, 10 exposures of 27 min each are taken, repeating the shift pattern twice, for a total exposure time of $4.5 \mathrm{~h}$. The typical layout of spectra on each CCD of the 4 channels is presented in Fig. 3 for the "VVDS-Deep".

\subsection{Observed fields}

The list of "VVDS-Deep" fields observed from October to December 2002 is presented in Table 2. A total of 20 pointings have been observed in the VVDS-Deep area of the VVDS-02h field and 5 pointings in the VVDS-CDFS data (Le Fèvre et al. 2004a). We have observed 30\% of the "Deep" area pointings during this period. The first epoch observations of the VVDS-Wide survey will be presented elsewhere (Garilli et al., in preparation).

\section{Pipeline processing of VIMOS multi-slit data with VIPGI}

\subsection{VIPGI: the VIMOS Interactive Pipeline Graphical Interface}

The pipeline processing of the VVDS data is performed using the VIMOS Interactive Pipeline Graphical Interface (VIPGI, see Scodeggio et al. 2005 for a full description). This pipeline is built from the same individual $\mathrm{C}$ code routines developed by the VIRMOS consortium and delivered to ESO, but was made autonomous so as not to be dependent on the ESO data environment.

\subsection{Spectra location}

The location of slits is known from the mask design process, hence, knowing the grism zero deviation wavelength and the dispersion curve, the location of spectra is known a priori on the detectors. However, small shifts from predicted positions are possible due to the complete manufacturing and observation process. From the predicted position, the locations of the observed spectra are identified accurately on the 4 detectors and an extraction window is defined for each slit/spectrum.

\subsection{Wavelength calibration}

The calibration in wavelength is secured by the observation of helium and argon arc lamps through the observed masks. Wavelength calibration spectra are extracted at the same location as the object spectra and calibration lines are identified to derive the pixel to wavelength mapping for each slit. The wavelength to detector pixel transformation is fit using a third order polynomial, resulting in a mean deviation $\sim 1 \AA$ rms across the wavelength range (Scodeggio et al. 2005).

\subsection{Sky subtraction, fringing correction}

This critical step in the data processing is handled at two levels. First, a low order polynomial (second order) is fit along the slit, representing the sky background contribution at each wavelength position, and subtracted from the $2 \mathrm{D}$ spectrum. 
Table 2. First epoch VVDS-Deep fields observations in the VVDS-02h and VVDS-CDFS.

\begin{tabular}{|c|c|c|c|}
\hline Field & $\alpha_{2000}$ & $\delta_{2000}$ & Exposure time (min) \\
\hline$F 02 P 016$ & $02 \mathrm{~h} 25 \mathrm{~m} 34.49 \mathrm{~s}$ & $-04 \operatorname{deg} 40^{\prime} 15.6^{\prime \prime}$ & $10 \times 27$ \\
\hline$F 02 P 017$ & $02 \mathrm{~h} 26 \mathrm{~m} 00.83 \mathrm{~s}$ & $-04 \operatorname{deg} 40^{\prime} 15.6^{\prime \prime}$ & $10 \times 27$ \\
\hline$F 02 P 018$ & $02 \mathrm{~h} 26 \mathrm{~m} 27.16 \mathrm{~s}$ & $-04 \operatorname{deg} 40^{\prime} 15.6^{\prime \prime}$ & $10 \times 27$ \\
\hline$F 02 P 019$ & $02 \mathrm{~h} 26 \mathrm{~m} 53.50 \mathrm{~s}$ & $-04 \operatorname{deg} 40^{\prime} 15.6^{\prime \prime}$ & $10 \times 27$ \\
\hline$F 02 P 020$ & $02 \mathrm{~h} 27 \mathrm{~m} 19.83 \mathrm{~s}$ & $-04 \operatorname{deg} 40^{\prime} 15.6^{\prime \prime}$ & $10 \times 27$ \\
\hline$F 02 P 027$ & $02 \mathrm{~h} 25 \mathrm{~m} 34.47 \mathrm{~s}$ & $-04 \operatorname{deg} 32^{\prime} 37.6^{\prime \prime}$ & $13 \times 27$ \\
\hline$F 02 P 028$ & $02 \mathrm{~h} 26 \mathrm{~m} 00.80 \mathrm{~s}$ & $-04 \operatorname{deg} 32^{\prime} 37.6^{\prime \prime}$ & $10 \times 27$ \\
\hline$F 02 P 029$ & $02 \mathrm{~h} 26 \mathrm{~m} 27.13 \mathrm{~s}$ & $-04 \operatorname{deg} 32^{\prime} 37.6^{\prime \prime}$ & $9 \times 24+5 \times 27$ \\
\hline$F 02 P 030$ & $02 \mathrm{~h} 26 \mathrm{~m} 53.46 \mathrm{~s}$ & $-04 \operatorname{deg} 32^{\prime} 37.6^{\prime \prime}$ & $10 \times 27$ \\
\hline$F 02 P 031$ & $02 \mathrm{~h} 27 \mathrm{~m} 19.79 \mathrm{~s}$ & $-04 \operatorname{deg} 32^{\prime} 37.6^{\prime \prime}$ & $9 \times 27$, except channel $2,8 \times 27$ \\
\hline$F 02 P 038$ & $02 \mathrm{~h} 25 \mathrm{~m} 34.45 \mathrm{~s}$ & $-04 \operatorname{deg} 24^{\prime} 59.6^{\prime \prime}$ & $11 \times 27$, except channel $3,4 \times 27$ (not used) \\
\hline$F 02 P 039$ & $02 \mathrm{~h} 26 \mathrm{~m} 00.77 \mathrm{~s}$ & $-04 \operatorname{deg} 24^{\prime} 59.6^{\prime \prime}$ & $10 \times 27$ \\
\hline$F 02 P 040$ & $02 \mathrm{~h} 26 \mathrm{~m} 27.10 \mathrm{~s}$ & $-04 \operatorname{deg} 24^{\prime} 59.6^{\prime \prime}$ & $11 \times 27$ \\
\hline$F 02 P 041$ & $02 \mathrm{~h} 26 \mathrm{~m} 53.42 \mathrm{~s}$ & $-04 \operatorname{deg} 24^{\prime} 59.6^{\prime \prime}$ & $10 \times 27$ \\
\hline$F 02 P 042$ & $02 \mathrm{~h} 27 \mathrm{~m} 19.75 \mathrm{~s}$ & $-04 \operatorname{deg} 24^{\prime} 59.6^{\prime \prime}$ & $7 \times 27$, except channel $2,2 \times 27$ not used \\
\hline$F 02 P 043$ & $02 \mathrm{~h} 27 \mathrm{~m} 46.07 \mathrm{~s}$ & $-04 \operatorname{deg} 24^{\prime} 59.6^{\prime \prime}$ & $12 \times 27$ \\
\hline$F 02 P 050$ & $02 \mathrm{~h} 26 \mathrm{~m} 00.74 \mathrm{~s}$ & $-04 \operatorname{deg} 17^{\prime} 21.6^{\prime \prime}$ & $10 \times 27$ \\
\hline$F 02 P 051$ & $02 \mathrm{~h} 26 \mathrm{~m} 27.06 \mathrm{~s}$ & -04 deg $17^{\prime} 21.6^{\prime \prime}$ & $10 \times 27$ \\
\hline$F 02 P 052$ & $02 \mathrm{~h} 26 \mathrm{~m} 53.39 \mathrm{~s}$ & -04 deg 17'21.6" & $10 \times 27$, except channel 2 : no data \\
\hline$F 02 P 053$ & $02 \mathrm{~h} 27 \mathrm{~m} 19.71 \mathrm{~s}$ & $-04 \operatorname{deg} 17^{\prime} 21.6^{\prime \prime}$ & $10 \times 27$ \\
\hline CDFS 001 & $03 \mathrm{~h} 32 \mathrm{~m} 28.0 \mathrm{~s}$ & $-27^{\circ} 48^{\prime} 30^{\prime \prime}$ & $10 \times 27$ \\
\hline CDFS 002 & $03 \mathrm{~h} 32 \mathrm{~m} 37.04 \mathrm{~s}$ & $-27^{\circ} 50^{\prime} 30^{\prime \prime}$ & $8 \times 27$ \\
\hline CDFS 003 & $03 \mathrm{~h} 32 \mathrm{~m} 18.95 \mathrm{~s}$ & $-27^{\circ} 50^{\prime} 30^{\prime \prime}$ & $9 \times 27$ \\
\hline$C D F S 004$ & $03 \mathrm{~h} 32 \mathrm{~m} 37.04 \mathrm{~s}$ & $-27^{\circ} 46^{\prime} 30^{\prime \prime}$ & $12 \times 27$ \\
\hline$C D F S 005$ & $03 \mathrm{~h} 32 \mathrm{~m} 18.95 \mathrm{~s}$ & $-27^{\circ} 46^{\prime} 30^{\prime \prime}$ & $10 \times 27$ \\
\hline
\end{tabular}

All exposures of a sequence ( 5 for the "Wide", 10 for the "Deep" survey) are then combined with a 3-sigma clipping algorithm. As the object is moved to different positions along the slit following the offset pattern, the median of the 2D sky subtracted spectra produces a frame from which the object is eliminated, but that includes all residuals not corrected by sky subtraction, in particular the fringing pattern varying with position across the slit and wavelength. This sky/fringing residual is then subtracted from each individual 2D sky subtracted frame; these are shifted following the offset pattern to register the object at the same position, and the individual frames are combined with a median, sigma-clipping (usually $2 \sigma$ ), algorithm to produce the final summed, sky subtracted, fringing corrected, 2D spectrum.

\subsection{D wavelength and flux calibrated spectra}

The last step done automatically by VIPGI is to extract a 1D spectrum from the summed 2D spectrum, using an optimum extraction following the slit profile measured in each slit (Horne 1986). The 1D spectrum is then flux calibrated using the ADU to absolute flux transformation computed from the observations of spectrophotometric standard stars. We have used the star LTT3218 to perform the flux calibration.

A final check of the 1D calibrated spectra is performed and the most discrepant features are removed manually, cleaning each spectrum of e.g. zero order contamination or negative nonphysical features.

\subsection{Spectrophotometric accuracy}

The quality of the spectrophotometry calibration can be estimated by a comparison of integrated magnitudes computed from the flux calibrated spectra, with the broad band photometric measurements coming from the deep imaging data. The comparison shown in Fig. 4 is done in absolute terms, comparing the $I$-band photometric magnitude with the $I$-band spectroscopic magnitude derived from the integration of the flux of spectra in the equivalent of the $I$-band photometric bandpass. The spectroscopic $I$-band magnitude is about 0.2 mag fainter than the photometric magnitude on average, increasing to about 0.5 mag fainter at the bright end of the survey. This can be directly associated with the slit losses inherent to 1 arcsec-wide slits placed on galaxies with sizes larger than the slit width.

The second quality check performed is the comparison of the photometric multi-color magnitudes with the flux in spectra, over the full spectroscopic wavelength range. It is apparent in Fig. 5 that the relative spectrophotometry is accurate to $\sim 10 \%$ over the wavelength range 5500-9500.

\subsection{Spectra signal to noise}

The signal to noise ratio, measured per spectral resolution element of $1000 \AA$ centered on $7500 \AA$, is shown in Fig. 6 for the VVDS-Deep. At the faintest magnitudes, the $\mathrm{S} / \mathrm{N}$ goes down to a mean of $\sim 4$. This $\mathrm{S} / \mathrm{N}$ is sufficient to enable redshift measurements while at the same time allowing the observation of a large number of objects as shown in Sects. 6 and 7. 


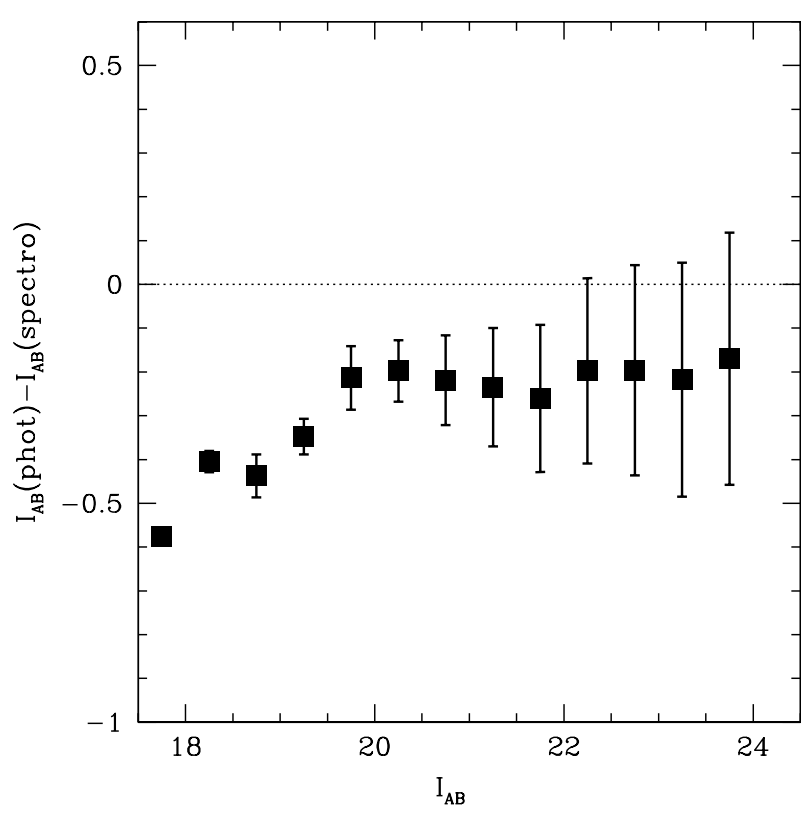

Fig. 4. Difference between the photometric $I$ band magnitude and the I-band magnitude computed from $\sim 1000$ calibrated VVDS spectra. The difference is mainly due to slit losses; no systematic effect in the spectrophotometric calibration is observed vs. magnitude.
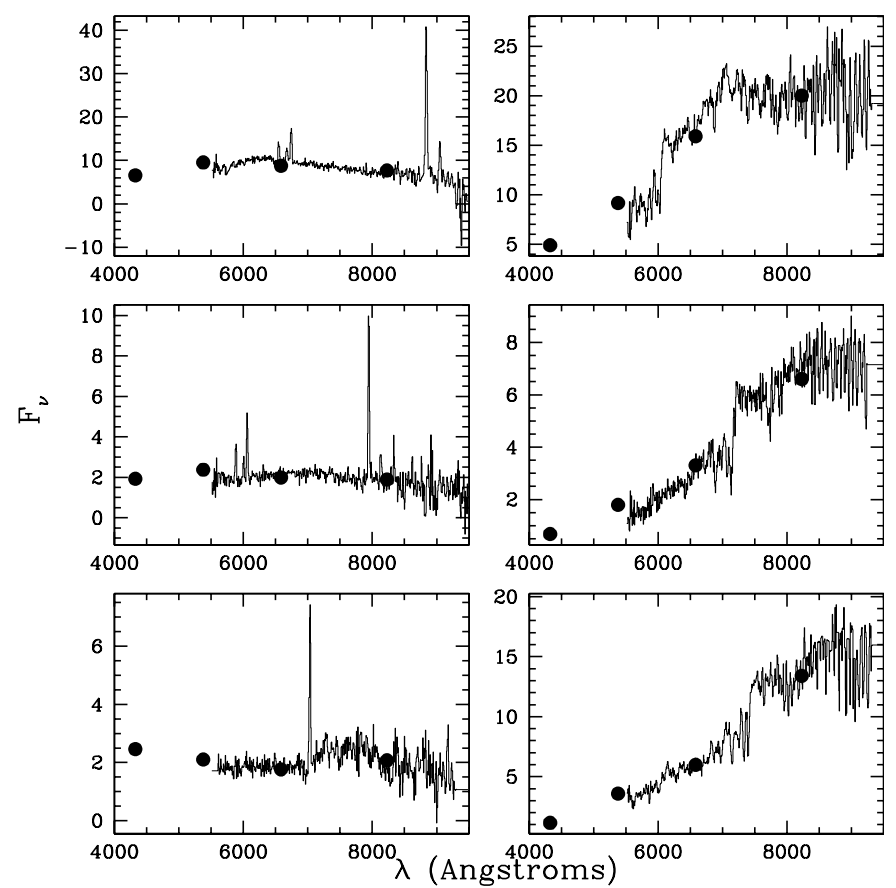

Fig. 5. Flux-calibrated VVDS spectra compared to the photometry of the VVDS imaging survey after scaling to match to the $I$ band flux (black dots). The spectrophotometric accuracy is conserved over the full wavelength coverage at the $10 \%$ level.

\section{Measuring redshifts}

\subsection{Building up experience over a large redshift base}

The VVDS is the first survey to assemble a complete spectroscopic sample of galaxies based on a simple $I$-band limit down to $I_{A B}=24$, spanning the redshift range $0 \leq z \leq \sim 5$. The spectral resolution $R \sim 230$ is adequate to measure redshifts

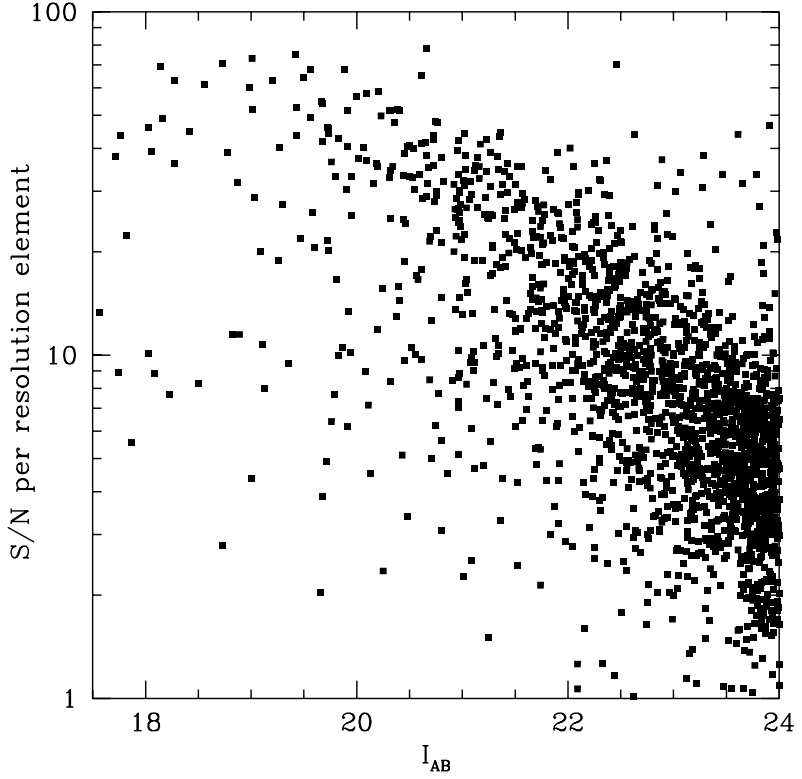

Fig. 6. $\mathrm{S} / \mathrm{N}$ per spectral resolution element over the magnitude range of the VVDS-Deep survey.

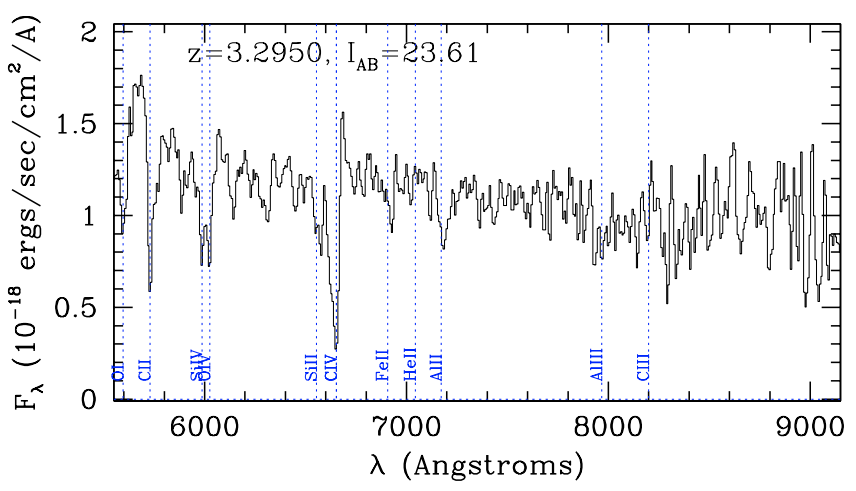

Fig. 7. Spectrum of an absorption line galaxy with $I_{A B}=23.61$, and $z=3.2950$, demonstrating the ability of low spectral resolution $R \sim$ 230 to measure redshifts from absorption line galaxies down to the very faint end of the survey.

of absorption line galaxies down to the faintest magnitudes observed, as shown in Fig. 7. Other examples of spectra are given in Sect. 7.6.

Magnitude-limited samples have the advantage of a controlled bias in the selection of the target galaxies, which can lead to a secure census of the galaxy population as seen at a given rest-frame wavelength (see e.g. Lilly et al. 1995b). The drawback is that, as the magnitudes become fainter, the redshift range becomes larger, and identifying redshifts out of a very large range of possibilities becomes increasingly harder from a fixed set of observed wavelengths. The VVDS wavelength range for the VIMOS observations is 5500-9500 ̊. It allows a secure follow-up of the spectral signature of galaxies longward of [OII] $3727 \AA$ and minimizes the bias in the identification of galaxy redshifts up to $\sim 1.5$.

A further difficulty in measuring redshifts in the VVDS is that no star/galaxy selection is done a priori before spectroscopy, hence a significant fraction of stars is included in the target list and they have to be identified in the redshift 
measurement process. Redshift measurements are thus considerably more challenging than in local surveys or for targeted high redshift surveys for which the redshift range is known from an a priori imposed selection function (e.g. Lyman-break galaxies, EROs, Lyman- $\alpha$ emission objects).

In addition, measuring the redshifts of galaxies with $1.5<z<2.7$ is quite challenging within the wavelength domain 5500-9500 $\AA$ that has generally faint features, and a lack of published observed galaxy templates in the range 1700-3000 $\AA$ to be used in cross-correlation programs such as the KBRED environments developed for the VVDS (Scaramella et al., in preparation). A dedicated approach had to be followed to ensure sufficient knowledge of rest-frame spectra with VIMOS, especially in the UV between $\sim 1700 \AA$ and $[\mathrm{OII}] 3727$ where previously observed spectra are not documented in the literature at the $\mathrm{S} / \mathrm{N}$ level required to be used as reference templates. These templates have been combined with the templates available below $1700 \AA$ from the observations of Lyman-break galaxies (Shapley et al. 2003) to produce templates covering 1100-8000 A rest frame.

\subsection{Crossing the "redshift desert"}

The "redshift desert" has been referred to as the redshift domain between the low-intermediate redshifts measured up to $z \sim 1.3-1.5$ and the high redshift galaxies identified using the Lyman-break technique, with a paucity of galaxies identified at $z \sim 1.5-2.7$. Crossing this "redshift desert" is critical to reduce the incompleteness of deep redshift surveys and to probe the galaxy population at an important time in the evolution.

In a first pass of redshift measurements on the first epoch spectra of the VVDS $I_{A B}=24$ sample (Le Fèvre et al. 2003b), $\sim 10 \%$ of galaxy redshifts appeared hard to identify while a significant fraction of these spectra had a good continuum $\mathrm{S} / \mathrm{N}$. We have established an iterative approach to identify these objects based on the creation of high $\mathrm{S} / \mathrm{N}$ rest-frame galaxy templates from observed VVDS spectra, going as far as possible toward the UV and bridging the gap between the well observed wavelength range above [OII] $3727 \AA$ and the range below $1700 \AA$ well represented by the templates assembled from $z \sim 3$ Lyman-break galaxies (Shapley et al. 2003). These new templates have been included in our redshift measurement engine KBRED and tested against the parent samples of galaxies used to produce them, in the range up to redshift $z=1.5$, then up to redshifts $z=2$ and above up to $z \sim 5$ as new redshifts were identified.

This approach has proved successful and has allowed us to identify a large population of objects with $z>1.5$ as shown in Sects. 8 and 9. Other groups have also recently efficiently explored this "redshift desert" (Abraham et al. 2004; Steidel et al. 2004). This "desert" is now demonstrated to be only the result of the selection function imposed by the combination of the faintness of the sources, the wavelength domain of observations, prior lack of observed galaxy templates and the strong $\mathrm{OH}$ sky emission features. In effect, the "VVDS redshift desert" is $2.2 \leq z \leq 2.7$, mainly associated with the combination of the wavelength domain of spectroscopic observations and the strong $\mathrm{OH}$ sky lines.

A complete account of the templates, the methods to identify the galaxies with $z \geq 1.5$, and the remaining incompleteness of the VVDS in the "redshift desert" is given elsewhere (Paltani et al., in preparation).

\subsection{Manual vs. automated redshift measurements with KBRED}

With templates covering the rest-frame range 1100-8000 $\AA$, two complementary approaches have been followed to measure redshifts: a manual approach and a computer-aided approach with the KBRED automated redshift measuring engine (Scaramella et al., in preparation).

KBRED is a set of routines implemented in the IDL programming environment to perform cross-correlation of observed spectra with reference star and galaxy templates. It includes advanced features to perform Principal Component Analysis of the spectra, projected on a base of reference templates, which are combined to reproduce the spectral energy distribution and measure the best redshift. KBRED can be run automatically on a list of spectra, or manually on a particular spectrum (see Scaramella et al., in preparation, for details).

The first step of redshift measurement has been to run the KBRED routine automatically on all 1D spectra, sky corrected and flux calibrated. This process correctly identifies about $60 \%$ of the redshifts, the main difficulty being that, despite intensive testing and code development, it has not been possible to come up with a single objective criterion or set of criteria capable of identifying a posteriori on which spectra KBRED has succeeded or failed to measure a redshift. This is due to the non-linear noise present in the spectra, mostly coming from CCD fringing correction residuals which can be strong above $8000 \AA$, making the computation of the classical cross-correlation strength parameter unreliable. Further developments of KBRED are in progress to solve this difficulty.

The second step in redshift measurement is then to visually examine objects one by one in the VIPGI environment, which makes available the $1 \mathrm{D}$ and 2D corrected spectra, object profile, sky emission and a set of tools to determine redshifts by hand. The redshift found by the automatic run of KBRED is displayed with the main spectral features expected superimposed on the 1D spectrum. For $\sim 60 \%$ of the spectra the user has simply to quickly verify that the KBRED redshift indeed corresponds to real spectral features and validate the measurement. For the other $\sim 40 \%$ of the spectra, the user notices that KBRED has identified a spectral feature that is noise, as determined upon examination of the $2 \mathrm{D}$ spectrum, based on the residuals of the sky subtraction at the location of the strong sky emission $\mathrm{OH}$ bands, compounded by the $\mathrm{CCD}$ fringing. A visual examination of the spectrum is carried out to mark the secure features, removing the strongest sky-noise features, and either run the VIPGI redshift calculator matching the marked features, or again run KBRED on the cleaned spectrum. 


\subsection{Checking the redshift measurements}

We use teams of data reducers to measure redshifts. For each pointing set of 4 VIMOS quadrants, one person performed the VIPGI processing from raw data to $2 \mathrm{D}$ and $1 \mathrm{D}$ sky corrected and calibrated spectra. The redshift measurement and quality flag assignment was then performed independently by two persons, and cross-checked together to solve the discrepant measurements. The performance of the team in determining the type of spectra, the spectral features to expect and the instrument signatures (e.g. fringing) increased significantly after the final stage of building a reference set of well-defined templates, as described above. Therefore, a last check of the measurements was performed by a third person, using the latest set of templates, before they were validated and entered in the VVDS database. During this last "super-check" the original value was ultimately changed for about $10 \%$ of the spectra.

Although time consuming, this procedure ensures that minimal machine or personal biases propagate throughout the survey. The redshift measurements and associated quality flags enable a statistical treatment of the overall quality of the survey, as described below.

\subsection{Quality flags}

We have used a classification of redshift quality similar to the scheme used in the Canada-France Redshift Survey (Le Fèvre et al. 1995):

- flag 4: a completely secure redshift, obvious spectral features in support of the redshift measurement;

- flag 3: a very secure redshift, strong spectral features;

- flag 2: a secure redshift measurement, several features in support of measurement;

- flag 1: a tentative redshift measurement, weak spectral features including continuum shape;

- flag 0: no redshift measurement, no apparent features;

- flag 9: one secure single spectral feature in emission, redshift assigned to [OII] $3727 \AA$, or $\mathrm{H} \alpha$, or in very rare cases to Ly $\alpha$.

A similar classification is used for broad line AGN: when one emission line is identified as "broad" (resolved at the spectral resolution of the VVDS), flags $11,12,13,14,19$ are used to identify the redshift quality. At this stage, no attempt has been made to separate starburst galaxies from type 2, narrow line AGN.

When a second object appears by chance in the slit of the main target, these objects are classified with a "2" added in front of the flag, leading to flags 20, 21, 22, 23, 24, 29. It is important to identify these objects separately from the main sample, as a significant fraction of their flux could be blocked by the slit because it has been centered on the main target, hence reducing the $\mathrm{S} / \mathrm{N}$ and redshift measurement ability for a given magnitude.

We have classified objects in slits with a clear observational problem as flag $=-10$, like e.g. objects for which the automated spectra detection algorithm in VIPGI failed, or objects too close to the edge of a slit to allow for a proper sky subtraction. This concerns less than $2 \%$ of the slits.
The flag number statistics for the VVDS-Deep sample on the CDFS and VVDS-02h is given in Table 3. The redshift distribution of flag 2 objects reasonably agrees with the overall redshift distribution as shown in Fig. 8, but only with a $44 \%$ probability that the two populations are drawn from the same sample (as indicated by a KS test), with significant differences between the two populations for $z>1.2$. The redshift distribution of flag 1 objects is significantly different, with a KS test indicating that this population has only a $7 \%$ probability of being drawn from the same sample as galaxies with flags 2, 3, and 4 . Flag 1 objects are predominantly at $z \geq 1.2$ as shown in Fig. 9.

We can estimate the probability of the redshift measurements of being correct for each of the quality flags in two ways. First we have compared the difference in redshift for the 426 objects observed twice in independent observations: we find a concordance in redshift within $\mathrm{d} z \leq 0.0025$, of $f c=31 \pm 7 \%$ for flags $1,65 \pm 8 \%$ for flags $2,94 \pm 8 \%$ for flags 3, and $99 \pm 7 \%$ for flags 4 . Assuming that the intrinsic probability of being correct for a given flag is a constant $p_{\text {flag }}$, then the fraction of concordant redshifts with the same flag is $p_{\text {flag }}^{2}$. From the fraction of concordant redshifts reported above, we therefore find $p_{\text {flag }}=f c^{0.5}$, or $p_{\text {flag }}=0.55,0.81,0.97,0.995$ for flags $1,2,3$, and 4 , respectively.

A second approach is to compare the spectroscopic redshifts for the whole sample to photometric redshifts derived from the photometric data (Bolzonela et al., in preparation). To obtain a large number of comparisons for galaxies with bright magnitudes $17.5 \leq I_{A B} \leq 22$, we have computed photometric redshifts for the full spectroscopic sample from the $B V R I$ photometric data. The $z_{\text {phot }}$ vs. $z_{\text {spec }}$ comparison in the VVDS-02h field is shown in Fig. 10. In addition, to obtain better constraints on photometric redshifts at faint magnitudes $22 \leq I_{A B} \leq 24$, we have computed photometric redshifts for the smaller sample for which $K$ photometry is available, as shown in Fig. 11. From the brightest objects in Fig. 10 and assuming that the flag 4 spectroscopic redshifts are $100 \%$ secure, we can identify an intrinsic error of about $5 \%$ in the photometric redshift measurements. Removing this fraction of failed photometric redshifts, we deduce that the spectroscopic flag 3 galaxies are $\sim 96 \%$ correct, $\sim 84 \%$ for flag 2 , while the "bright" flag 1 would be $\sim 58 \%$ correct. Using the faintest galaxies in Fig. 11, we similarly deduce that the faint galaxies with flags 3,2 , and 1 are $\sim 91 \%, \sim 81 \%$, and $\sim 48 \%$ correct, respectively. We thus determined that objects with quality flags $1,2,3$, and 4 , are $48-58 \%, \sim 81 \%$, $91-97 \%$ and $\sim 99 \%$ correct, respectively. This is in agreement with the values derived from the repeated spectroscopic observations as reported above.

\section{The "First epoch" VVDS-deep sample}

\subsection{Galaxies, stars, and QSOs}

A total of 10157 galaxies have a spectroscopically measured redshift, 8591 for primary targets with flags $\geq 2$, and an additional 1566 with flag 1 . An additional 278 galaxies with flags $\geq 2$ and 83 with flag 1 have been measured as secondary targets appearing by chance in the slit of a primary target. 
Table 3. Statistics of redshift quality flags for the First Epoch VVDS-Deep sample.

\begin{tabular}{|c|c|c|c|c|c|c|c|c|c|c|c|c|c|c|c|c|c|c|c|c|c|c|c|c|c|}
\hline Field & 0 & 1 & 2 & 3 & 4 & 9 & 11 & 12 & 13 & 14 & 19 & 20 & 21 & & 222 & 232 & 24 & 29 & 211 & 212 & 21 & 132 & 214 & 219 & Total \\
\hline CDFS & 102 & 140 & 506 & 480 & 285 & 115 & 1 & 6 & 1 & 2 & 0 & 20 & 10 & & $34 \quad 1$ & 10 & 9 & 1 & 0 & 0 & j & 0 & 0 & 0 & 1722 \\
\hline galaxies\&QSOs & - & 137 & 482 & 458 & 203 & 115 & 1 & 6 & 1 & 2 & 0 & - & -10 & & 31 & 9 & 5 & 1 & 0 & 0 & & 0 & 0 & 0 & 1461 \\
\hline stars & - & 3 & 24 & 22 & 82 & 0 & - & - & - & - & - & - & -0 & 0 & 3 & 1 & 4 & 0 & - & - & & - & - & - & 139 \\
\hline VVDS-02h & 690 & 1426 & 2557 & 2187 & 2157 & 304 & 13 & 17 & 27 & 8 & 10 & 149 & 73 & & 985 & 54 & 41 & 26 & 0 & 2 & & 2 & 0 & 1 & 9842 \\
\hline galaxies\&QSOs & - & 1363 & 2433 & 2069 & 1776 & 304 & 13 & 17 & 27 & 8 & 10 & 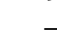 & -72 & & 945 & 523 & 37 & 26 & 0 & 2 & & 2 & 0 & 1 & 8306 \\
\hline stars & - & 63 & 124 & 118 & 381 & 0 & - & - & - & - & - & - & -1 & 1 & 4 & 2 & 4 & 0 & - & - & - & - & - & - & 697 \\
\hline Total & & & & & & & & & & & & & & & & & & & & & & & & & \\
\hline $\begin{array}{l}\text { VVDS-Deep } \\
1 \text { st epoch }\end{array}$ & 792 & 1566 & 3063 & 2667 & 2442 & 419 & 14 & 23 & 28 & 10 & 10 & 169 & 83 & & 32 & 64 & 50 & 27 & 0 & 2 & 2 & 2 & 0 & 1 & 11564 \\
\hline
\end{tabular}

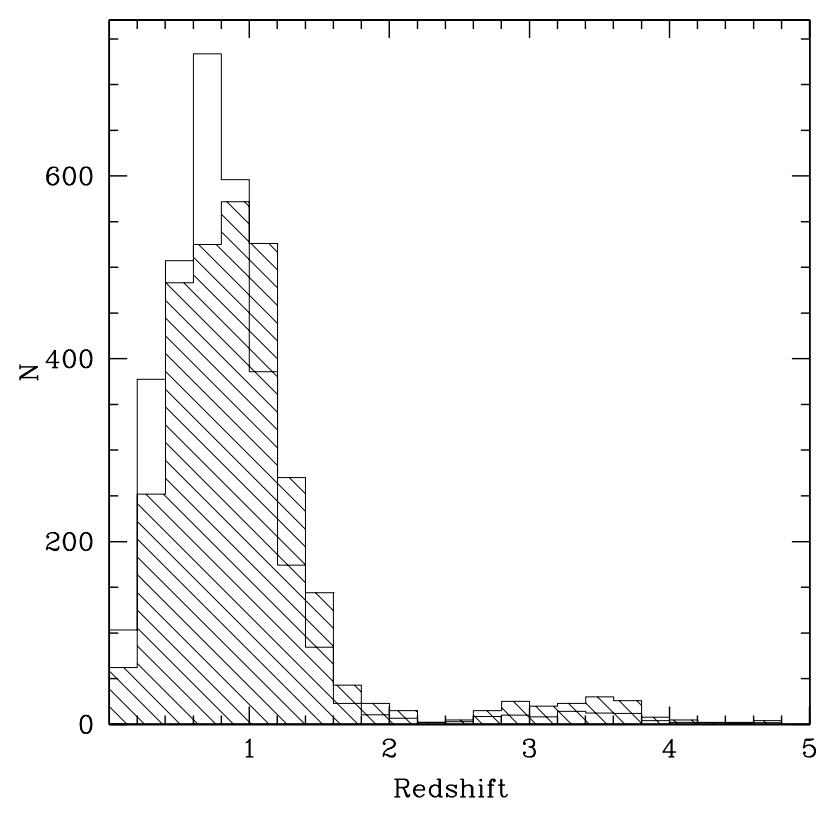

Fig. 8. Redshift distribution of flag 2 objects (dashed), compared to the normalized redshift distribution of the flag 3 and 4 objects (open).

A total of 836 stars have been spectroscopically measured in the VVDS-02h and the VVDS-CDFS (19 as secondary targets). This was expected since no a priori selection was made against compact objects in the photometric catalog.

There are 71 spectroscopically identified QSOs (flags 12, $13,14,19)$ ranging in redshift from $z=0.172$ to $z=3.863$, and an additional 14 QSOs with flag 11. This unique sample probes the faint end of the AGN luminosity function at high redshift, and will be described extensively in subsequent papers (Gavignaud et al.; Zamorani et al., in preparation).

\subsection{Spatial distribution of observed galaxies}

The spatial distribution of galaxies in the first epoch observations of the VVDS-02h field is shown in Fig. 12, for a total sampled area of $1750 \mathrm{arcmin}^{2}$. Together with the VVDS data obtained in a $453 \operatorname{arcmin}^{2}$ area around the CDFS (Le Fèvre et al. 2004b), a total $2203 \mathrm{arcmin}^{2}$, a $0.61 \mathrm{deg}^{2}$ area has been surveyed. This constitutes an unprecedented spectroscopic survey area at a depth as deep as $I_{A B}=24$.

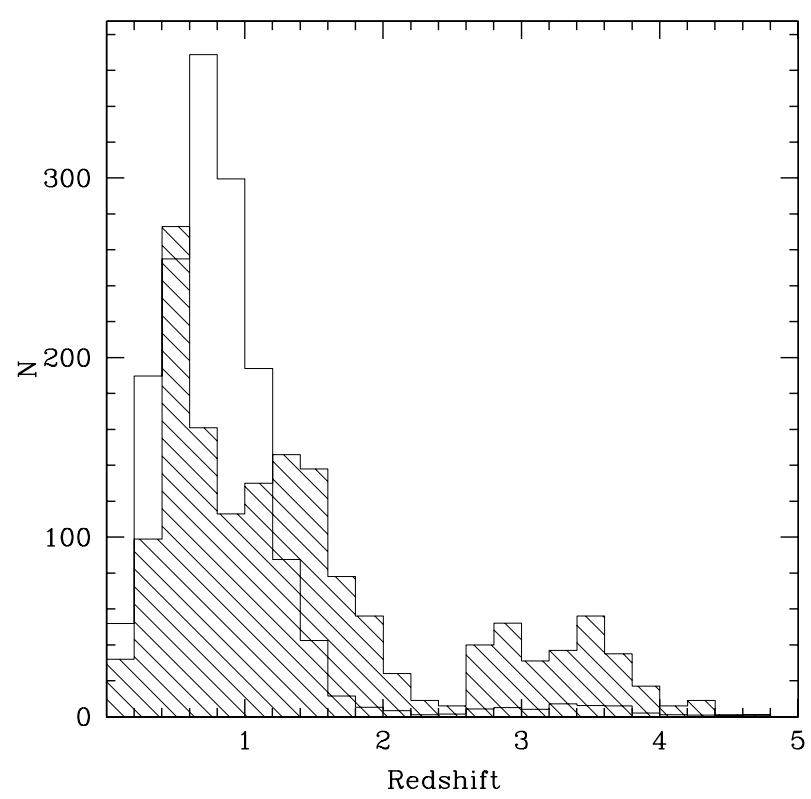

Fig. 9. Redshift distribution of flag 1 objects (dashed), compared to the normalized redshift distribution of the flag 2, 3 and 4 objects (open).

\subsection{Target sampling rate}

The current sampling of the $17.5 \leq I_{A B} \leq 24$ photometric sources by the VVDS is indicated in Fig. 13 for the VVDS-02h field, while the sampling in the VVDS-CDFS is almost constant at 30\% (Le Fèvre et al. 2004b). Spectra have been obtained in the VVDS-02h for a total of $22.8 \%$ of the photometric sources, averaged over the whole area observed, while in the central area corresponding to about two third of the field, 40\% of the photometric sources have been measured. The slit optimization technique used in VMMPS favors slit placement on smaller objects (Bottini et al. 2005), hence the ratio of spectroscopically observed objects to objects in the photometric catalog is not constant with magnitude for the VVDS-02h, while this optimization has not been used for the VVDS-CDFS.

\subsection{Redshift accuracy}

The accuracy of the redshift measurements can be estimated from the independent observations of the same objects either within the VVDS or with other instruments. We have observed 


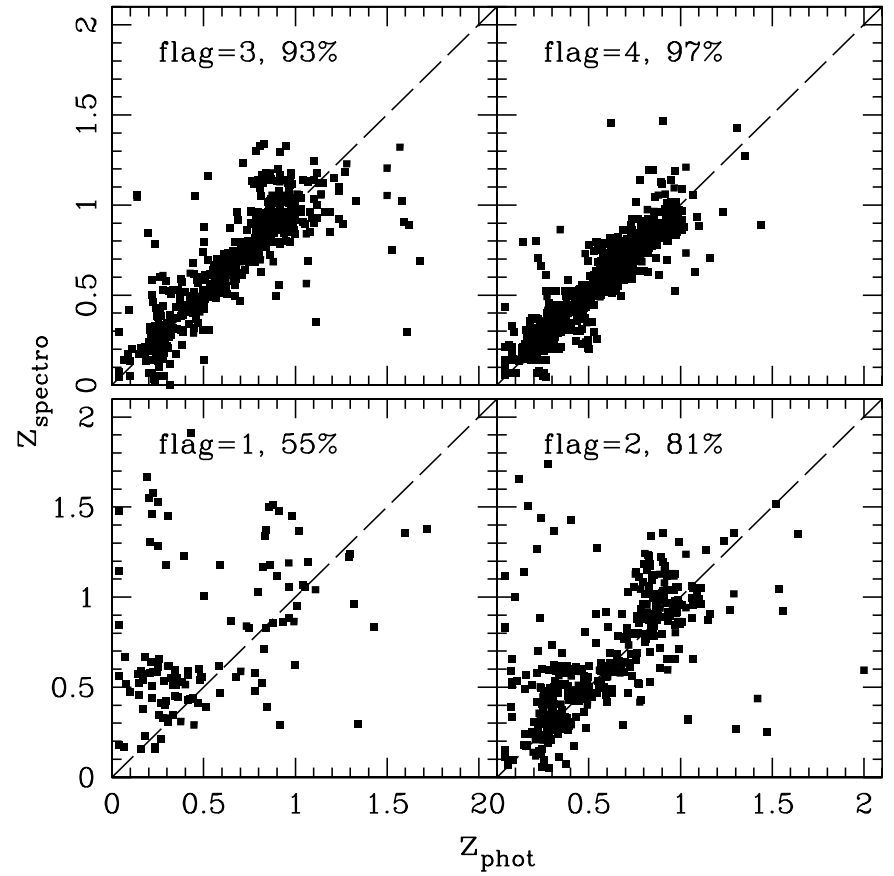

Fig. 10. Comparison of spectroscopic redshifts vs. photometric redshifts computed from $B V R I$ photometry for objects with magnitude $17.5 \leq I_{A B} \leq 22.5$, for each of the quality flags $1,2,3$ and 4 (from bottom left to top right). The fraction of galaxies for which the difference in photometric redshift vs. spectroscopic redshift is less than 0.2 is indicated on the top of each panel. Flags 3 and 4 represent very secure redshift measurements as indicated by the comparison of objects observed spectroscopically twice (see text), hence the dispersion observed for these flags is representative of the error in measuring photometric redshifts. For flags 2 and 1, errors from the photometric redshift estimate and the spectroscopic measurements combine to provide a lower value of $16 \%$ and $42 \%$ of discrepant redshifts, respectively.

160 objects twice in the CDFS fields (Le Fèvre et al. 2004b), and 266 objects twice in the VVDS-02h. The redshift difference between observations in the CDFS and in the VVDS-02h is plotted in Fig. 14; we find that the difference between two measurements has a Gaussian distribution with $\sigma_{z}=13 \times 10^{-4}$, or $\sim 390 \mathrm{~km} \mathrm{~s}^{-1}$, hence the accuracy of single redshift measurements is $390 /(2)^{0.5}$ or $276 \mathrm{~km} \mathrm{~s}^{-1}$. The 33 galaxies observed in common by VIMOS in the VVDS and FORS2 on the VLT by the GOODS team in the CDFS (2004) provide an external check of our measuring scheme. As shown in Fig. 15, the difference between the two measurements has a $\sigma_{z_{\mathrm{VVDS}}-z_{\mathrm{FORS}}}=$ $12.6 \times 10^{-4}, 378 \mathrm{~km} \mathrm{~s}^{-1}$, very similar to what is found from the repeat observations in the VVDS.

\subsection{Completeness vs. magnitude}

The completeness in redshift measurement is indicated in Fig. 16. Using only the best quality flags (flags 2, 3, 4, 9), the redshift measurement completeness is $78 \%$, while including the less secure flag 1 objects it reaches $93 \%$. The incomplete fraction translates into a sampling of the galaxy population which varies with galaxy type and redshift. This is modeled to compute statistical indicators like the luminosity function (Ilbert et al. 2005).

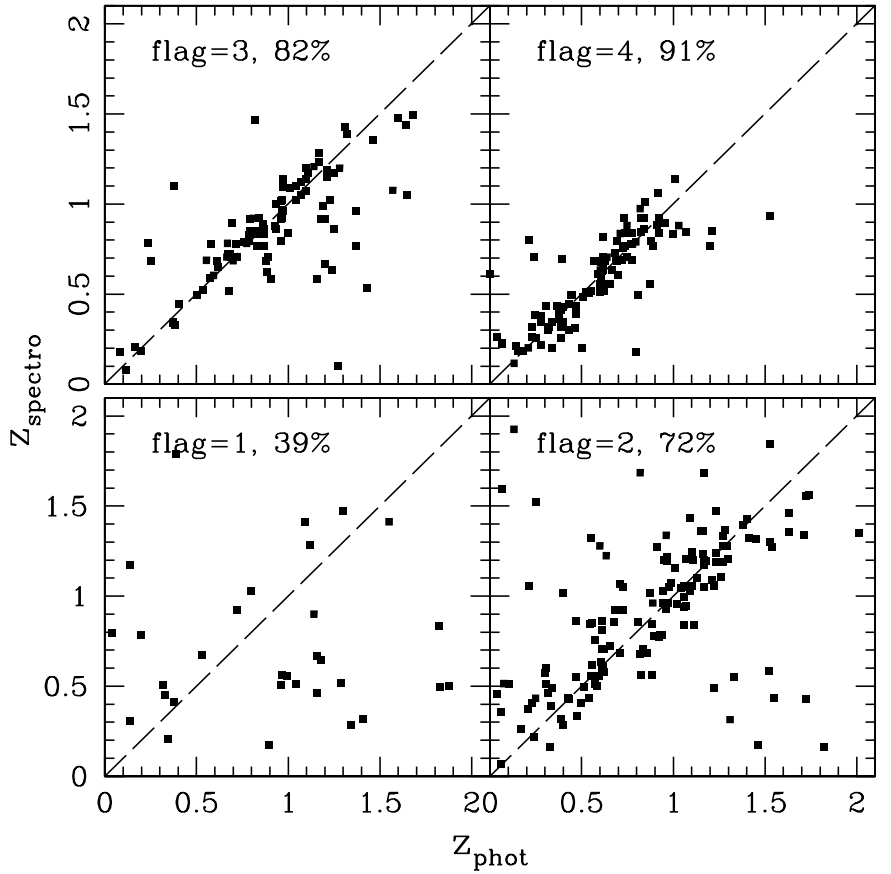

Fig. 11. Same as Fig. 10 for objects with magnitude $22 \leq I_{A B} \leq 24$, for each of the quality flags $1,2,3$ and 4 (from bottom left to top right), in the VVDS-02h area where $K$ band data is available in addition to $B V R I$ data and makes the photometric redshifts more accurate. The fraction of concordant redshifts is $38 \%, 72 \%, 82 \%$ and $91 \%$ for flags 1, 2, 3 and 4 respectively. Taking into account a photometric redshift failure of $\sim 9 \%$ as shown by the fraction of concordant flag 4 which are known to be $\sim 100 \%$ accurate from spectroscopy, we find that flags 1,2 and 3 are $47 \%, 81 \%, 91 \%$ correct, respectively, in this faint magnitude regime where the accuracy of photometric redshifts rapidly decreases.

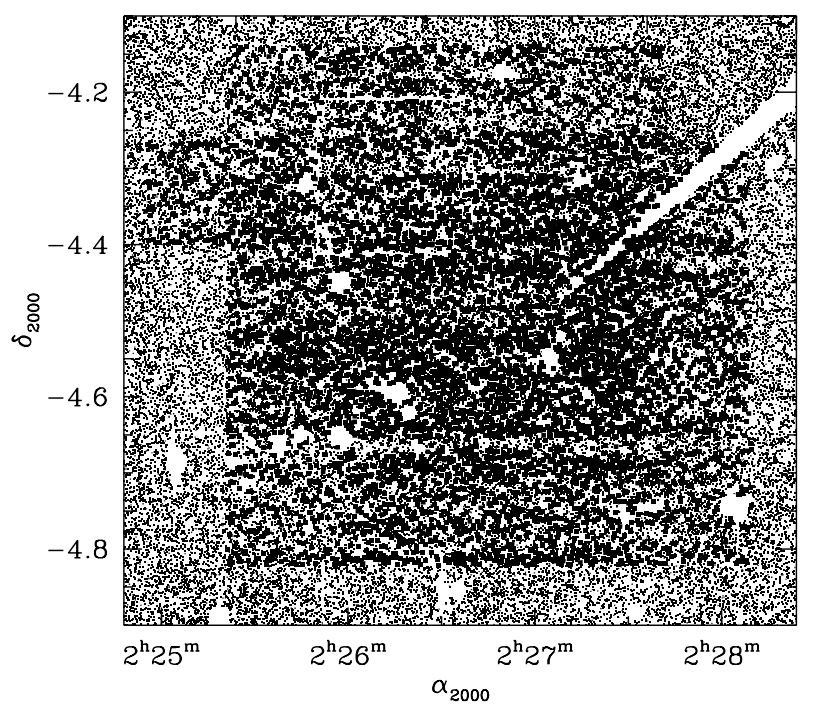

Fig. 12. Distribution of galaxies observed in VVDS-02h.

\subsection{Spectra}

Examples of spectra in the various redshift ranges are given in Figs. 17 to 20 . 


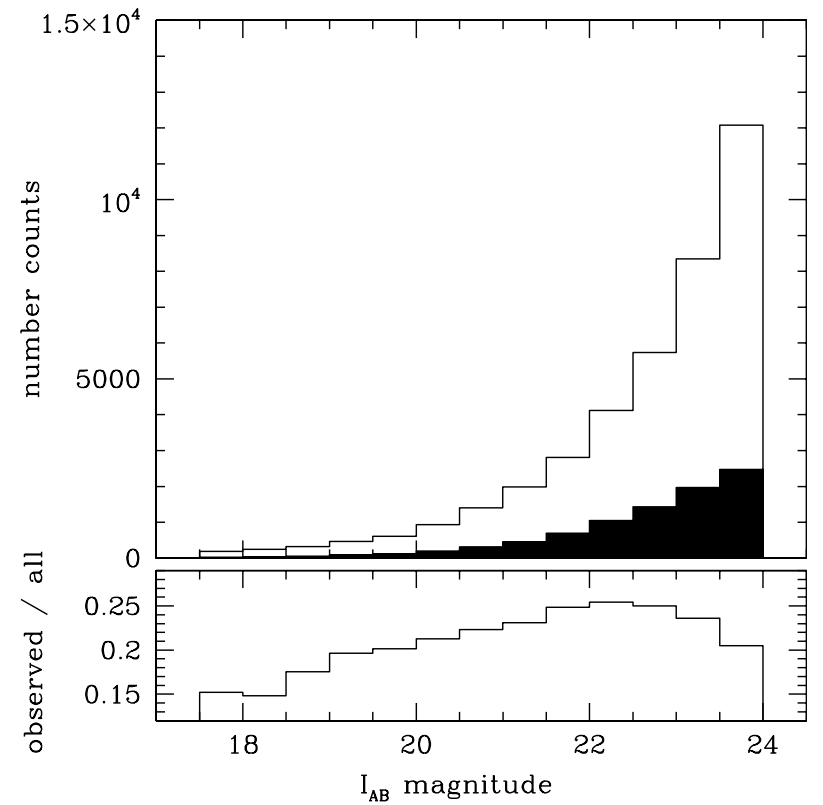

Fig. 13. Number and fraction of objects observed in the VVDS-02h, compared to the total number of objects in the photometric catalog with $17.5 \leq I_{A B} \leq 24$.

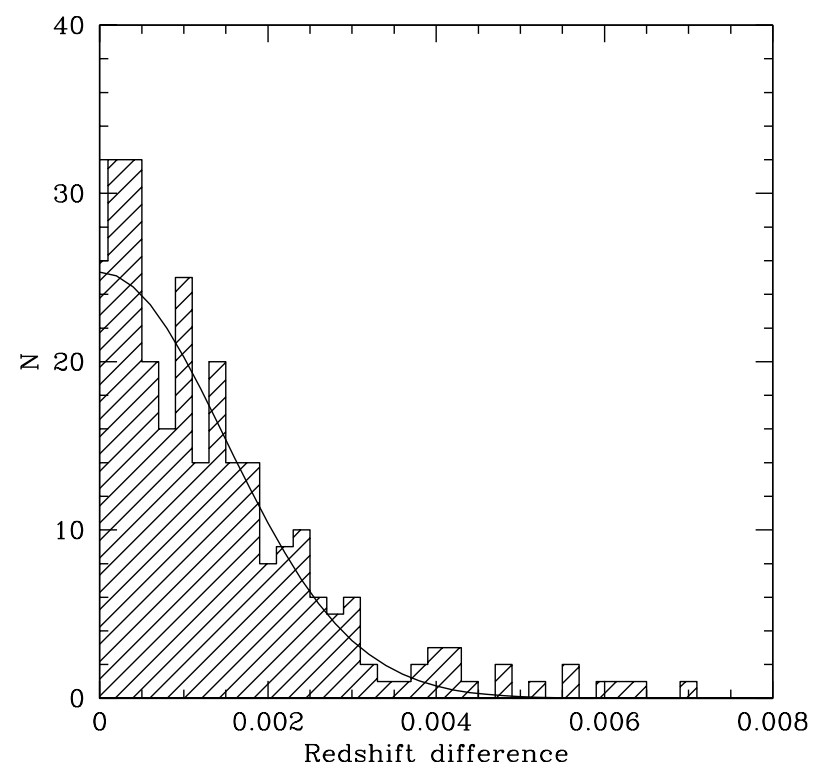

Fig. 14. Difference in redshift measurements for 266 galaxies observed twice in the VVDS-02h and 139 galaxies observed twice in the VVDS-CDFS. The difference has a Gaussian distribution with $\sigma_{z}=13 \times 10^{-4}$ or $390 \mathrm{~km} \mathrm{~s}^{-1}$, hence each single redshift measurement has an accuracy $390 /(2)^{0.5}$ or $276 \mathrm{~km} \mathrm{~s}^{-1}$.

\section{The galaxy population probed by the VVDS-Deep survey}

\subsection{Absolute magnitude distribution}

The absolute magnitude $M_{B_{A B}}$ distribution vs. redshift is presented in Fig. 21. At the lowest redshifts $z<0.4$, the VVDSDeep observes intrinsically faint galaxies with a mean $M_{B_{A B}} \sim$ -18 spanning a large range $-13.5 \leq M_{B_{A B}} \leq-21$. At intermediate redshifts $0.4<z<0.8$, galaxies are observed with a

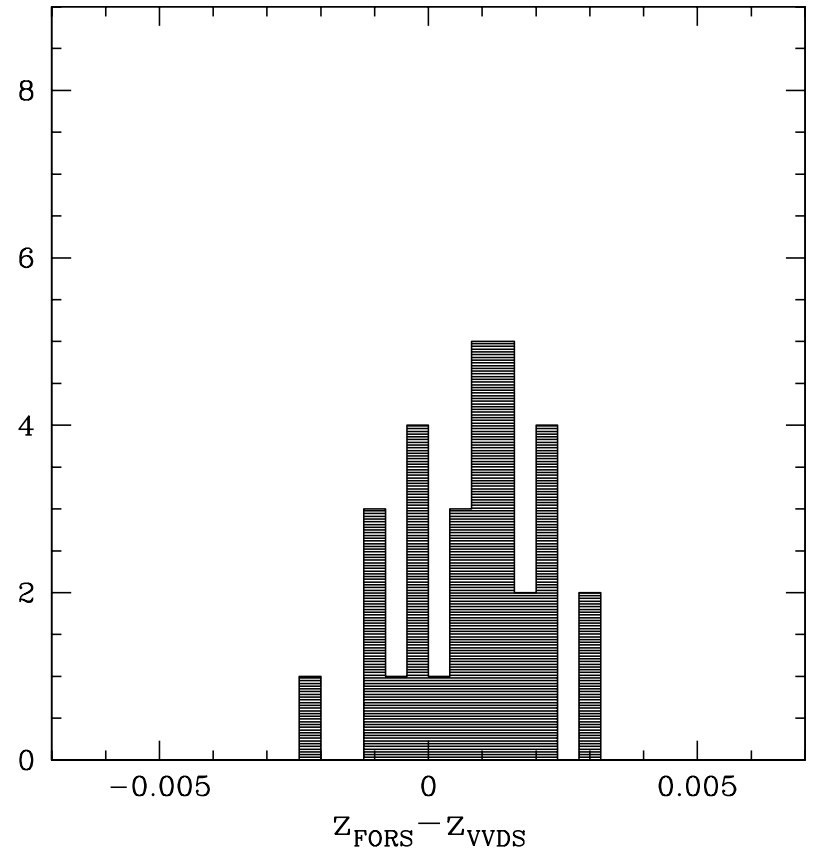

Fig. 15. Difference in redshift measurements for 33 objects in common between the VVDS-CDFS sample and the FORS2-GOODS observations of Vanzela et al. (2004). The difference has a mean $\mathrm{d} z=$ $8.4 \times 10^{-4}$ and a $\sigma_{z}=12.6 \times 10^{-4}$ or $378 \mathrm{~km} \mathrm{~s}^{-1}$.

mean $M_{B_{A B}} \sim-19.5$ in the range $-17 \leq M_{B_{A B}} \leq-22.5$, while for $1<z<2$ observed galaxies are brighter than $M_{B_{A B}} \sim-19$. This is a consequence of the pure apparent $I$-band magnitude selection, and the shift in the mean and range in absolute luminosity with redshift has to be taken into account when interpreting the observations. As the redshift range is large, the $k(z)$ corrections applied to transform apparent magnitudes to the rest frame $B$ absolute magnitude $M_{B_{A B}}$ work best for redshifts $z<1.5$ for which our broad band photometry can be used to constrain the rest frame $B$ luminosity. At $z>1.5$ the computation of $k(z)$ and $M_{B_{A B}}$ using template fitting becomes more uncertain, and UV rest absolute magnitudes are more appropriate (see Ilbert et al. 2005 for more details).

\subsection{Population at increasing redshifts to $z \sim 5$}

The number of galaxies in several redshift slices is given in Table 4. There are:

- 4004 objects with $z \leq 0.75$ and secure redshifts (4528 have measured redshifts when flag 1 objects are included);

- 3344 objects with $0.75<z \leq 1.4$ and secure redshifts (3744 have measured redshifts when flag 1 objects are included);

- 305 objects with $1.4<z \leq 2.5$ and secure redshifts (603 have measured redshifts when flag 1 objects are included);

- 186 objects with $2.5<z \leq 5.0$ and secure redshifts (462 have measured redshifts when flag 1 objects are included).

This constitutes the largest spectroscopic redshift sample over the redshift range $0.4 \leq z \leq 5$ assembled to date. A detailed account of the properties of the various populations probed by the VVDS-Deep will be given elsewhere (Paltani et al., in preparation). 


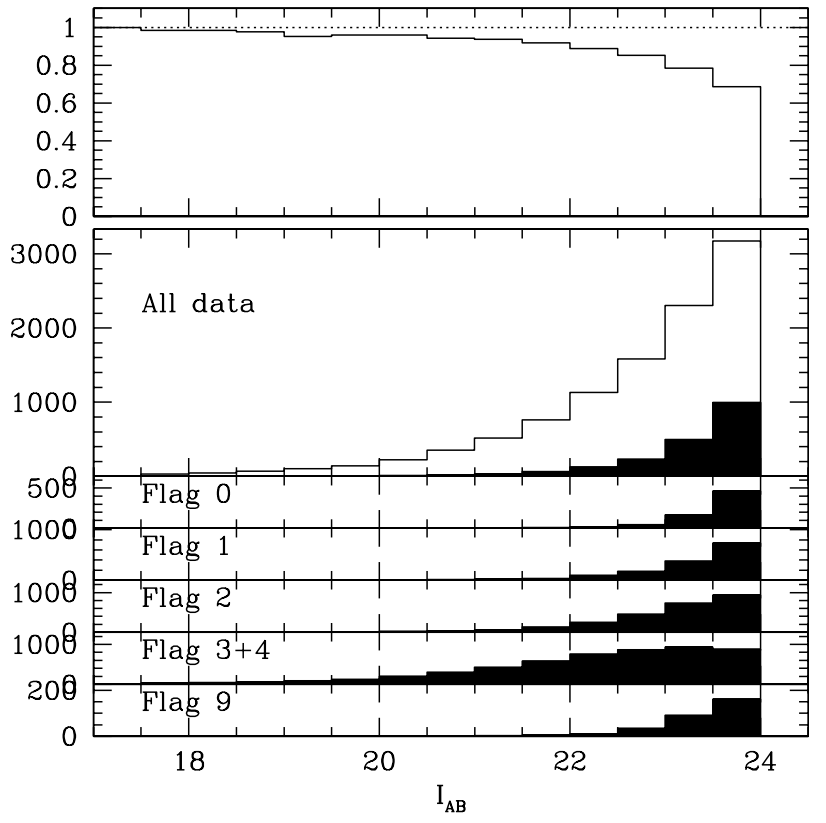

Fig. 16. Completeness of the $I_{A B} \leq 24$ sample in the VVDS- $02 \mathrm{~h}$ field. (Bottom panel) the magnitude distribution of galaxies with flags 0,1 , 2, 3+4 and 9; (central panel) the magnitude distribution of galaxies with all flags (open histogram), compared to the distribution of flags 1 and 2 (filled); (top panel) the ratio of secure redshift measurements with flags 2, 3, 4, 9 and of all measurements (filled). The overall redshift measurement completeness is $78 \%$ (flags $2,3,4,9$ ) and redshifts are measured for $93 \%$ of the sample (including flags 1).

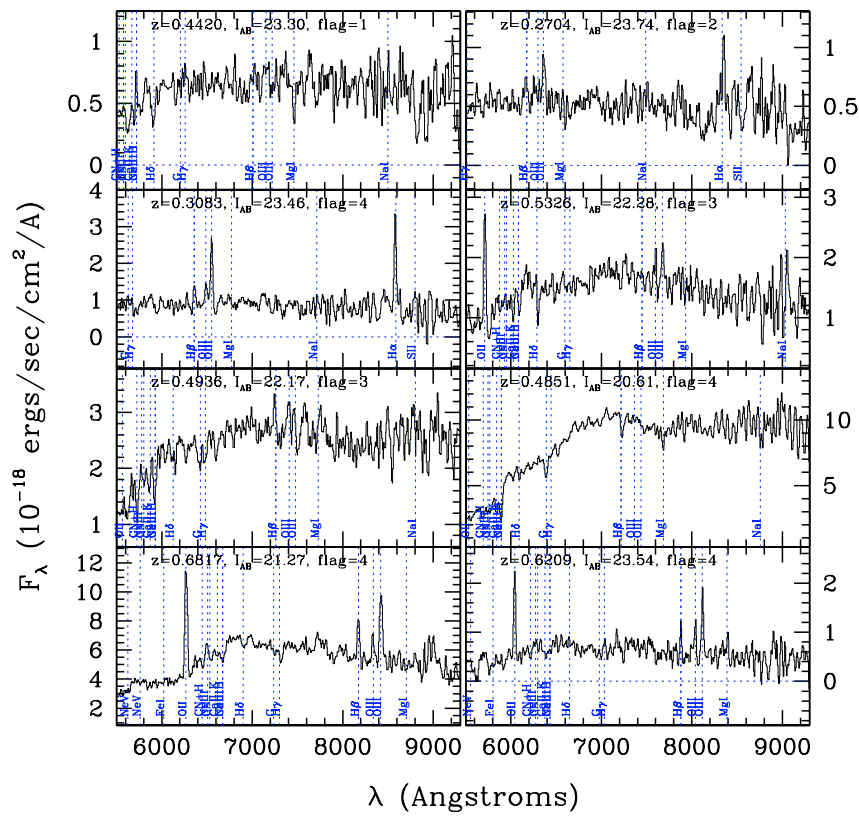

Fig. 17. VVDS spectra in the range $0<z \leq 0.7$.

\section{Redshift distribution for magnitude-limited samples at $I_{A B} \leq \mathbf{2 2 . 5}$ and $I_{A B} \leq 24$}

\subsection{Redshift distribution for $17.5 \leq I_{A B} \leq 22.5$, $17.5 \leq I_{A B} \leq 23$, and $17.5 \leq I_{A B} \leq 23.5$ magnitude limited samples}

From the $17.5 \leq I_{A B} \leq 24$ sample, we can extract samples magnitude-limited down to $I_{A B} \leq 22.5, I_{A B} \leq 23$, and

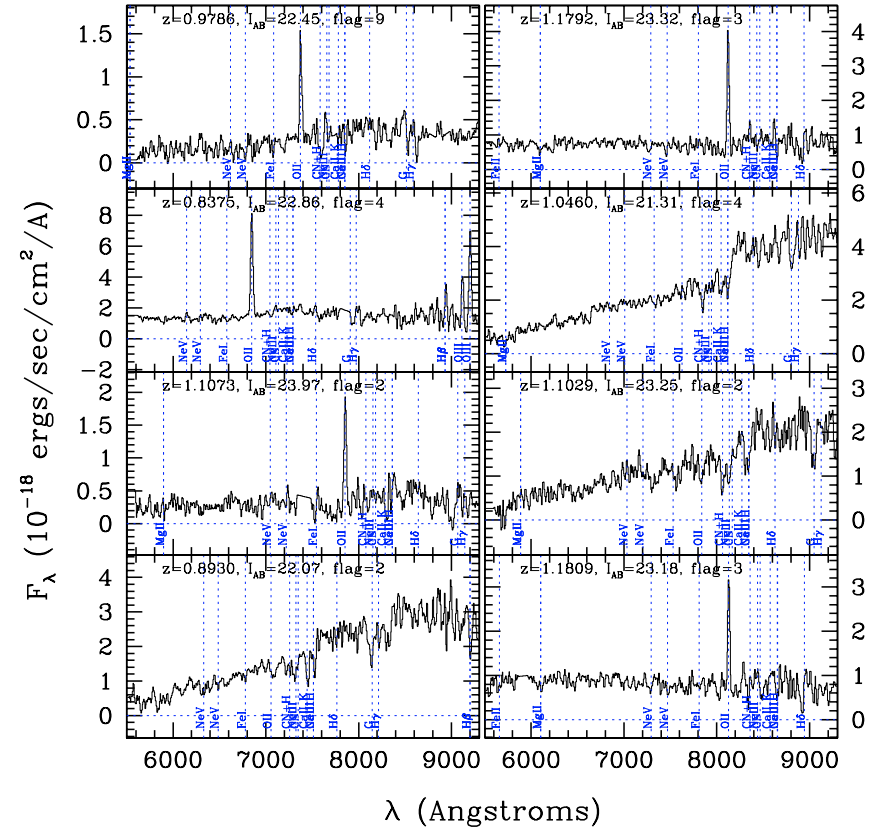

Fig. 18. VVDS spectra in the range $0.7<z \leq 1.3$.

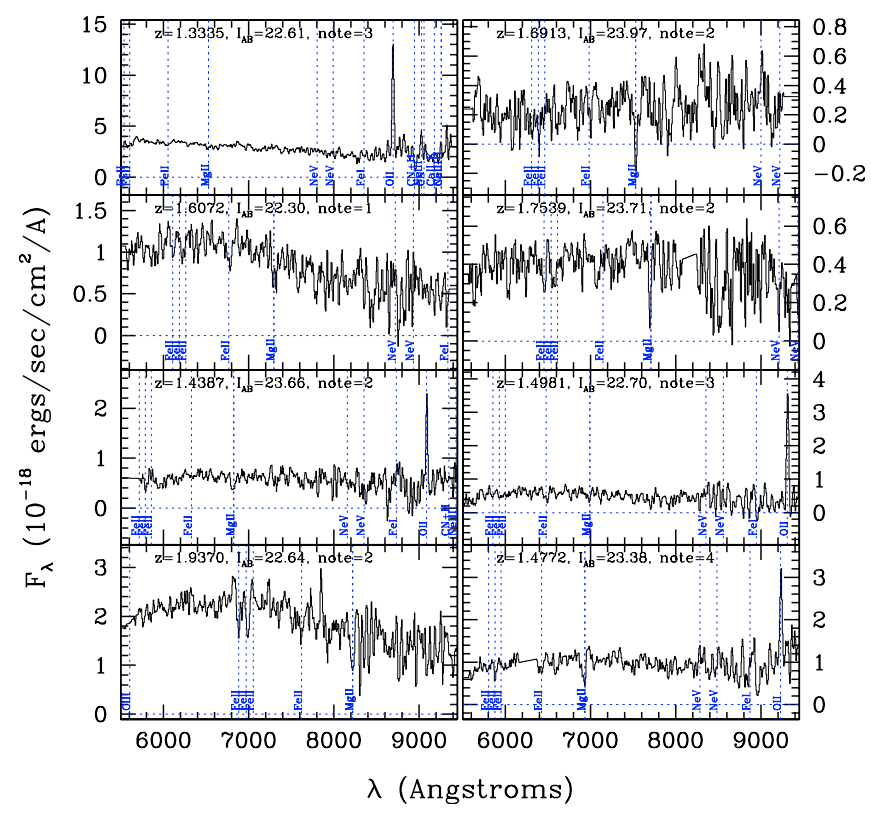

Fig. 19. VVDS spectra in the range $1.3<z \leq 2.2$.

$I_{A B} \leq 23.5$, which are $93 \%, 91 \%, 86 \%$ complete respectively including only flags 2, 3, 4 and $9(99 \%, 98 \%, 96 \%$ including objects with flags 1 ). The redshift distributions shown in Figs. 22 to 24 are therefore very secure. The redshift distribution shifts slightly to higher redshifts going from a median redshift of $z=0.62$ for $17.5 \leq I_{A B} \leq 22.5$, to $z=0.70$ for $17.5 \leq I_{A B} \leq 23.5$. The median redshift and 1st and 3rd quartiles are reported in Table 5 . There is only a marginal high redshift tail appearing at $z \geq 2$ in the faintest magnitude bin to $I_{A B}=23.5$. The redshift distribution for a sample down to $I_{A B} \leq 23.5$ does not go down significantly deeper in redshift than a sample limited to $I_{A B} \leq 22.5$, a fact to take into account when planning future redshift surveys. 


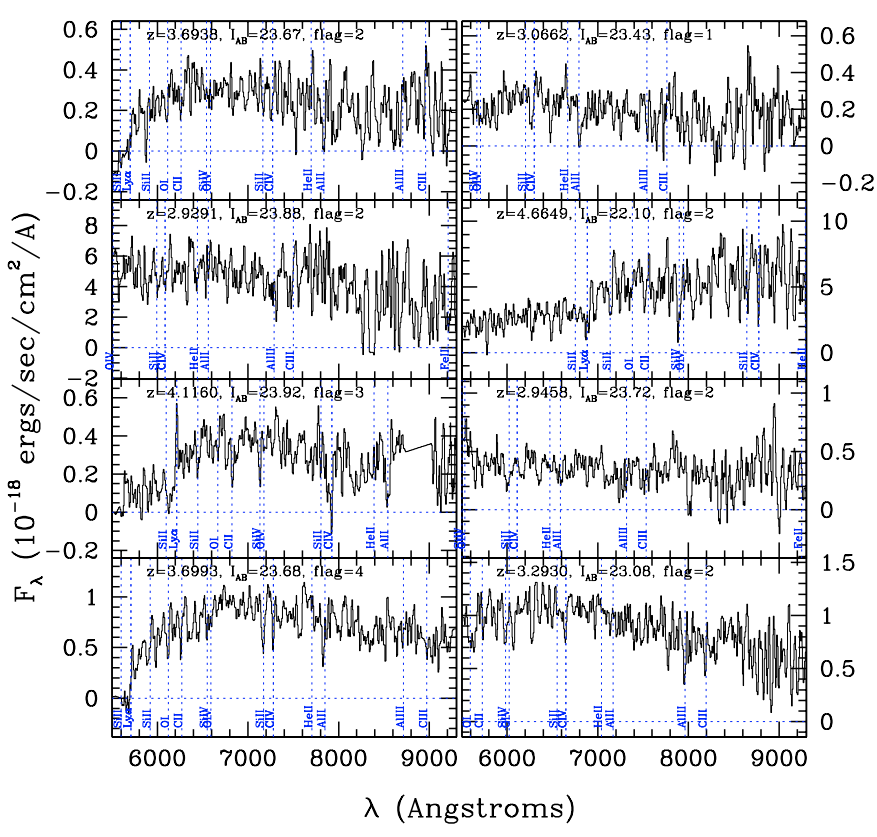

Fig. 20. VVDS spectra in the range $2.2<z \leq 5$.

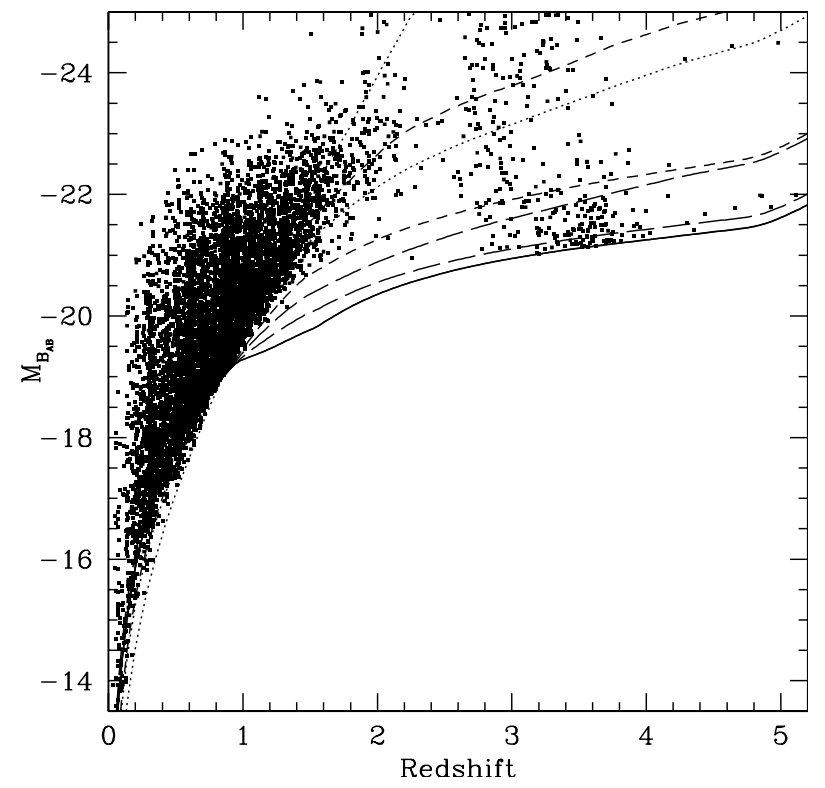

Fig. 21. Absolute $M_{B_{A B}}$ magnitude vs. redshift in VVDS-02h. The full sample is shown together with the tracks of CWW templates (Coleman et al. 1980) and starburst templates used to compute the $k(z)$ correction. At high redshift, the bluest tracks (starburst galaxies) correspond to the faintest absolute $M_{B}$ magnitudes. Each track has been normalized to produce $I_{A B}=24$ at all redshifts. As redshift increases, the computation of $k(z)$ for the rest frame $B$ band becomes increasingly difficult to constrain, hence produces a large range in $B$-band absolute luminosities for $z \geq 2$. At these high redshifts, and given the wavelength coverage of our spectroscopy and photometry, it is more appropriate to compute absolute magnitudes in the near UV.

\subsection{Redshift distribution for $17.5 \leq I_{A B} \leq 24$}

The redshift distribution of the full First Epoch VVDS-Deep sample is shown in Fig. 25. A total of 9340 primary target galaxies is present in this distribution including the VVDS-02h and VVDS-CDFS fields. The sample has a median redshift $z=0.76$, and a significant high redshift tail appears up to $z=5.123$. The $17.5 \leq I_{A B} \leq 24$ magnitude selection allows us to continuously sample the galaxy population at all redshifts. The VVDS spectroscopic measurement of the $N(z)$ distribution improves upon previous determinations using photometric redshifts (Brodwin et al. 2005), in particular in the range $1.4 \leq z \leq 5$ where the number of galaxies is small compared to the fraction of catastrophic failures generally encountered when computing photometric redshifts. This fraction of galaxies appearing at all redshifts $2<z<\sim 5$ indicates that the observations are deep enough to probe the brightest part of the population at these redshifts. The gap in the redshift distribution in the interval $2.2 \leq z \leq 2.7$ is readily understood in terms of a lower efficiency in measuring redshifts for galaxies in this range because of the VVDS observed wavelength range (see Sect. 6.2 and Paltani et al., in preparation). Objects with $z>2$ are examined in detail, in particular to evaluate the fraction of flag 1 (and to a much lesser extent, flag 2) galaxies lying at these redshifts; and the properties of this population will be discussed elsewhere (Paltani et al., in preparation).

\subsection{Redshift distribution in the VVDS-02h field}

The redshift distribution of galaxies in the VVDS-02h field is presented in Fig. 26 with smaller redshift bins. The field shows an alternance of strong density peaks and almost empty regions, with strong peaks identified all across the redshift range, although less prominent at $z>\sim 2$. This is the first time that the large-scale structure distribution of galaxies has been probed on transverse scales $\sim 30 \mathrm{~h}^{-1} \mathrm{Mpc}$ at these redshifts. A detailed analysis of the clustering properties of galaxies will be published elsewhere (Le Fèvre et al. 2005; Marinoni et al. 2005).

\section{Conclusion and summary}

We have presented the strategy and the first epoch data of the VVDS, an $I$-band limited deep redshift survey of the distant universe. We have been able to observe 11564 objects in the range $0 \leq z \leq 5.2$, in a total area of $\sim 2200 \operatorname{arcmin}^{2}$ in the VVDS-02h and VVDS-CDFS fields, which is thus the largest deep redshift survey at a depth $17.5 \leq I_{A B} \leq 24$ to date.

The multi-slit observations with the VLT-VIMOS instrument and heavy data processing with our VIPGI pipeline are described. Emphasis has been placed on the measurement of redshifts using the KBRED automated redshift measuring engine and the associated quality control. Independent redshift measurements have been produced and compared between two survey members, a final check was performed by a third party, and quality flags assigned to each redshift measurement. Repeated observations of the same objects in independent observations have allowed us to quantify the velocity accuracy of the survey as $276 \mathrm{~km} \mathrm{~s}^{-1}$. From these repeated measurements, and using photometric redshifts, we have been able to quantify the reliability of each of the redshift quality flags, and hence to derive the completeness of the survey in terms of the fraction of secure redshift measurements.

A total of 9340 redshifts have been measured on primary targets (7840 objects have the most secure flags 2, 3, 4 and 9), 
Table 4. Number of measured galaxies in redshift slices of the VVDS-Deep First Epoch (primary targets only).

\begin{tabular}{lcccccccc}
\hline \hline Field & $0-0.5$ & $0.5-0.75$ & $0.75-1$ & $1-1.4$ & $1.4-2.5$ & $2.5-3.5$ & $3.5-5$ & All $z$ \\
\hline CDFS, flag $\geq 1$ & 280 & 518 & 245 & 265 & 50 & 22 & 15 & 1395 \\
VVDS-02h, flag $\geq 1$ & 1674 & 2056 & 1801 & 1433 & 553 & 264 & 161 & 7945 \\
\hline $\begin{array}{l}\text { Total } \\
\text { VVDS-Deep }\end{array}$ & 1954 & 2574 & 2046 & 1698 & 603 & 286 & 176 & 9340 \\
1st epoch, flag $\geq 1$ & & & & & & & & \\
\hline $\begin{array}{l}\text { CDFS, flag } \geq 2 \\
\text { VVDS-02h, flag } \geq 2\end{array}$ & 259 & 490 & 223 & 243 & 28 & 8 & 7 & 1258 \\
\hline $\begin{array}{l}\text { Total } \\
\text { VVDS-Deep }\end{array}$ & 1766 & 1789 & 1688 & 1190 & 277 & 101 & 70 & 6582 \\
1st epoch, flag $\geq 2$ & 1725 & 2279 & 1911 & 1433 & 305 & 109 & 77 & 7840 \\
\hline
\end{tabular}

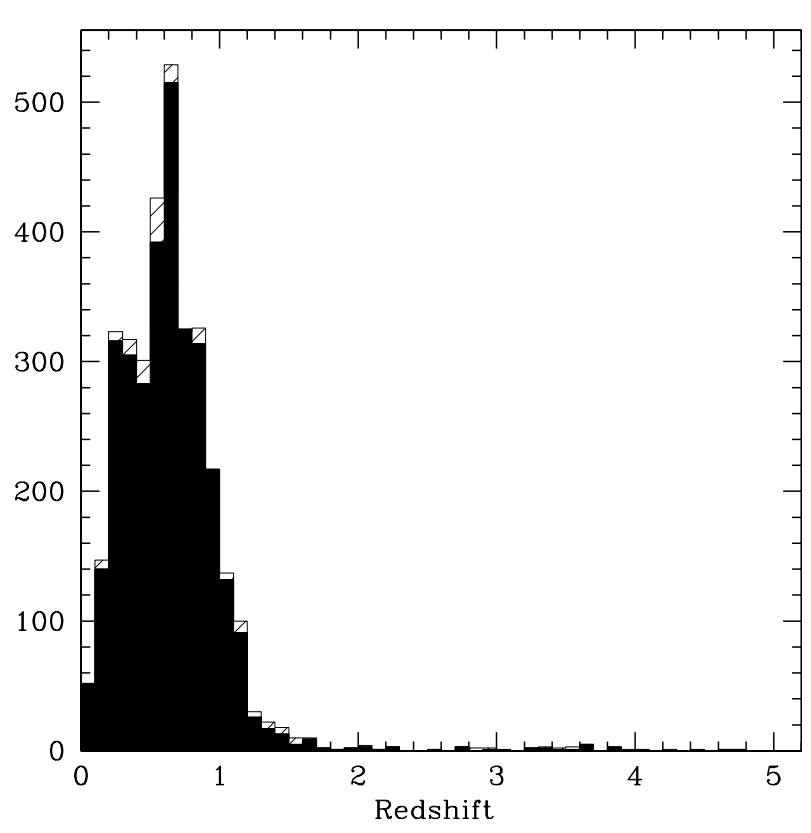

Fig. 22. Redshift distribution for a magnitude-limited sample of 3621 galaxies with $17.5 \leq I_{A B} \leq 22.5$, extracted from the sum of the VVDSCDFS and the VVDS-F02h fields. Galaxies with flags 2, 3, 4 and 9 are represented by the filled histogram, galaxies with flag 1 by the open histogram. This sample, including flags 2, 3, 4 and 9, is $93 \%$ complete. The median redshift is $z=0.62$ and the mean redshift is $z=0.65$.

and an additional 342 redshifts were obtained on secondary targets falling in the multi-slits by chance (of which 260 are the most secure). A total of 603 galaxies (305 with the most secure redshifts) have been measured in the range $1.4 \leq z \leq 2.5$, and 462 in the range $2.5 \leq z \leq 5.2$ (186 with the most secure redshifts). Without a priori compactness selection in the photometric catalog our spectroscopic sample includes 836 galactic stars, but 90 QSOs have been successfully identified. Following the quality flags associated with the redshift measurements, the sample is $78 \%$ complete (secure redshifts), while $93 \%$ of the sample has a redshift measured. We have presented the core properties of the sample in terms of spatial distribution, absolute magnitude and $B-I$ color vs. redshift, and presented examples of observed spectra, revealing the wide range of galaxy types and luminosities present in the survey.

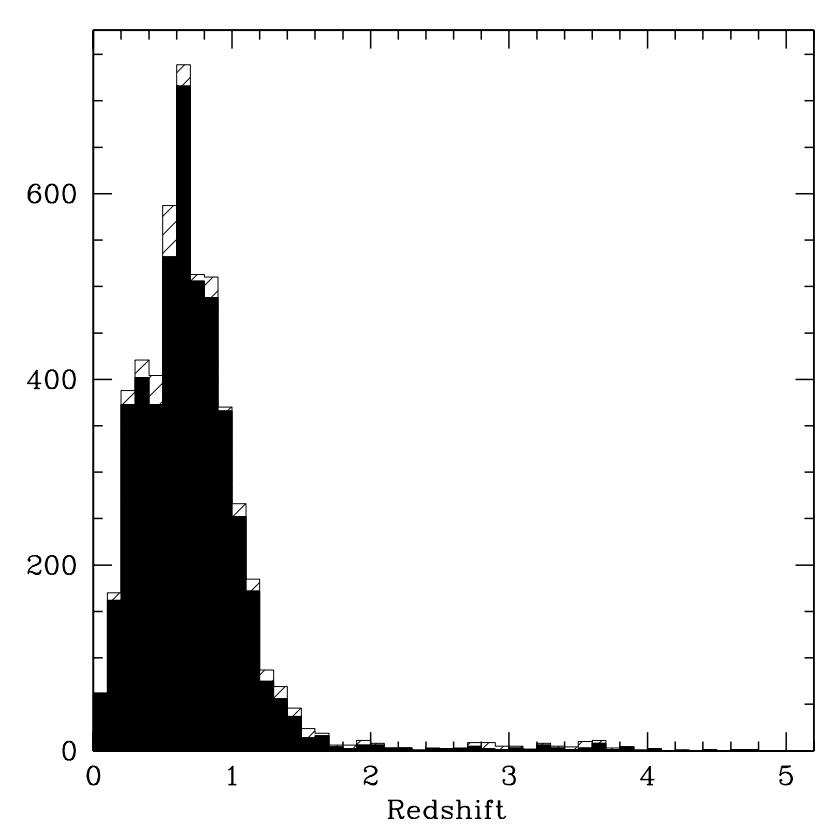

Fig. 23. Same as Fig. 22 for 5124 galaxies with $17.5 \leq I_{A B} \leq 23$. This sample, including flags 2, 3, 4 and 9 , is $90 \%$ complete. The median redshift is $z=0.67$ and the mean redshift is $z=0.72$.

We also presented the redshift distribution of magnitude limited samples down to $I_{A B}=24$. For samples purely selected in $I$-band magnitude, with $17.5 \leq I_{A B} \leq 22.5,17.5 \leq$ $I_{A B} \leq 23.0$ and $17.5 \leq I_{A B} \leq 23.5$, we find a median redshift of $z=0.62,0.67$ and 0.70 , respectively. For the complete first epoch $17.5 \leq I_{A B} \leq 24.0$ VVDS-Deep sample, we find that the median redshift is $z=0.76$, with a significant high redshift tail $1.5<z<5.2$ readily apparent.

The first epoch VVDS dataset presented here is used extensively by the VVDS team to measure evolution in the galaxy population, as presented in joint papers and several papers in preparation. It provides an unprecedented sample to study galaxy evolution over $90 \%$ of the life of the universe.

Acknowledgements. This research has been developed within the framework of the VVDS consortium (formerly the VIRMOS consortium).

This work has been partially supported by the CNRS-INSU and its Programme National de Cosmologie (France) and by Italian 


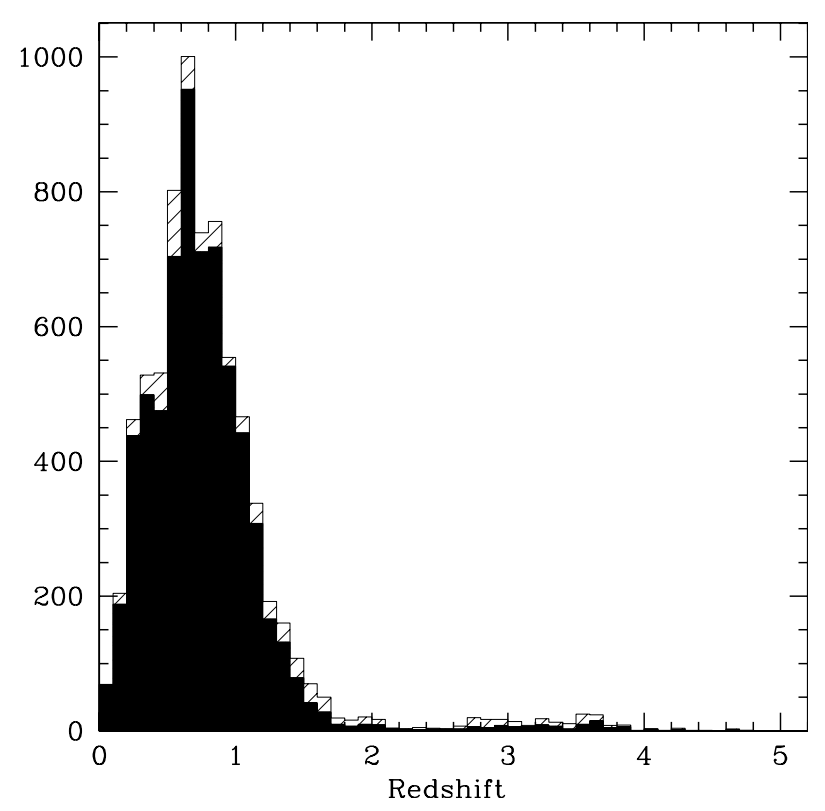

Fig. 24. Same as Fig. 22 for 7054 galaxies with $17.5 \leq I_{A B} \leq 23.5$. This sample, including flags $2,3,4$ and 9 , is $85 \%$ complete. The median redshift is $z=0.70$ and the mean redshift is $z=0.80$.

Table 5. Median redshifts, 1st and 2nd quartiles of the redshift distribution vs. magnitude (primary targets only).

\begin{tabular}{ccccc}
\hline \hline$I_{A B}$ range & $N_{\text {obj }}$ (flags $\left.\geq 2\right)$ & 1st quartile & Median & 3rd quartile \\
\hline $18.0-18.5$ & 9 & 0.13 & 0.18 & 0.24 \\
$18.5-19.0$ & 23 & 0.21 & 0.30 & 0.37 \\
$19.0-19.5$ & 45 & 0.21 & 0.30 & 0.41 \\
$19.5-20.0$ & 67 & 0.24 & 0.36 & 0.52 \\
$20.0-20.5$ & 111 & 0.26 & 0.43 & 0.64 \\
$20.5-21.0$ & 228 & 0.32 & 0.50 & 0.68 \\
$21.0-21.5$ & 361 & 0.38 & 0.58 & 0.76 \\
$21.5-22.0$ & 569 & 0.42 & 0.65 & 0.89 \\
$22.0-22.5$ & 833 & 0.46 & 0.70 & 0.91 \\
$22.5-23.0$ & 1156 & 0.50 & 0.79 & 1.11 \\
$23.0-23.5$ & 1493 & 0.56 & 0.85 & 1.17 \\
$23.5-24.0$ & 1675 & 0.58 & 0.90 & 1.32 \\
\hline
\end{tabular}

Research Ministry (MIUR) grants COFIN2000 (MM02037133) and COFIN2003 (No. 2003020150).

The VLT-VIMOS observations have been carried out on guaranteed time (GTO) allocated by the European Southern Observatory (ESO) to the VIRMOS consortium, under a contractual agreement between the Centre National de la Recherche Scientifique of France, heading a consortium of French and Italian institutes, and ESO, to design, manufacture and test the VIMOS instrument.

\section{References}

Abazajian, K., Adelman-McCarthy, J. K., Agueros, M. A., et al. 2003, AJ, 126, 2081

Abraham, R. G., Glazebrook, K., McCarthy, P. J., et al. 2004, AJ, 127 , 2455

Arnouts, S., Vandame, B., \& Benoist, C. 2001, A\&A, 379, 740

Arnouts, S., Schiminovich, D., Ilbert, O., et al. 2004, ApJ, 619, 43

Bertin, E., \& Arnouts, S. A\&AS, 124, 163

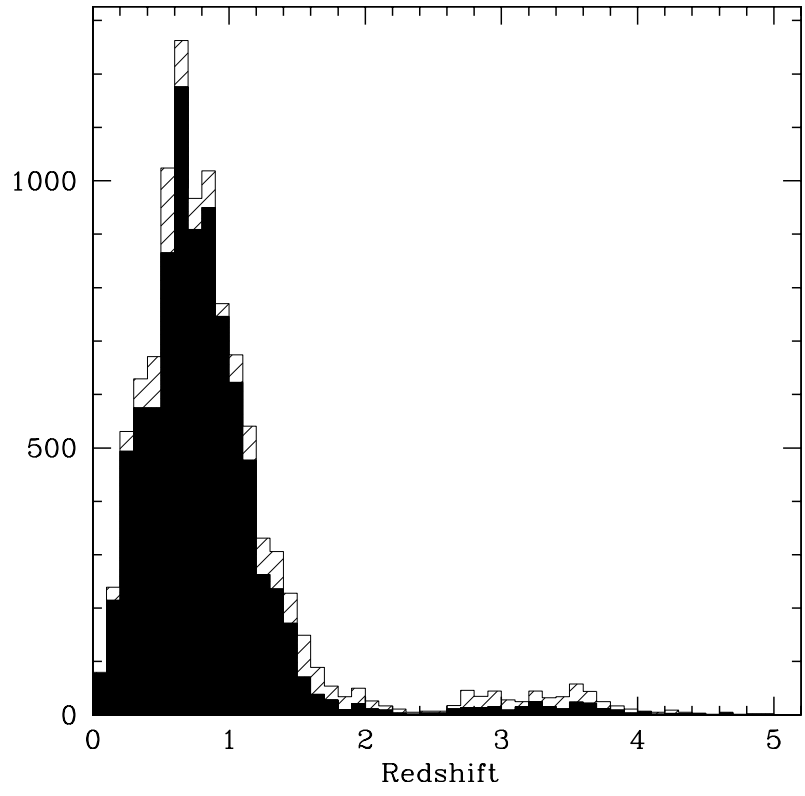

Fig. 25. Same as Fig. 22 for 9141 galaxies with $17.5 \leq I_{A B} \leq 24$. This sample, including flags 2, 3, 4 and 9 , is $78 \%$ complete. The median redshift is $z=0.76$ and the mean redshift is $z=0.90$.

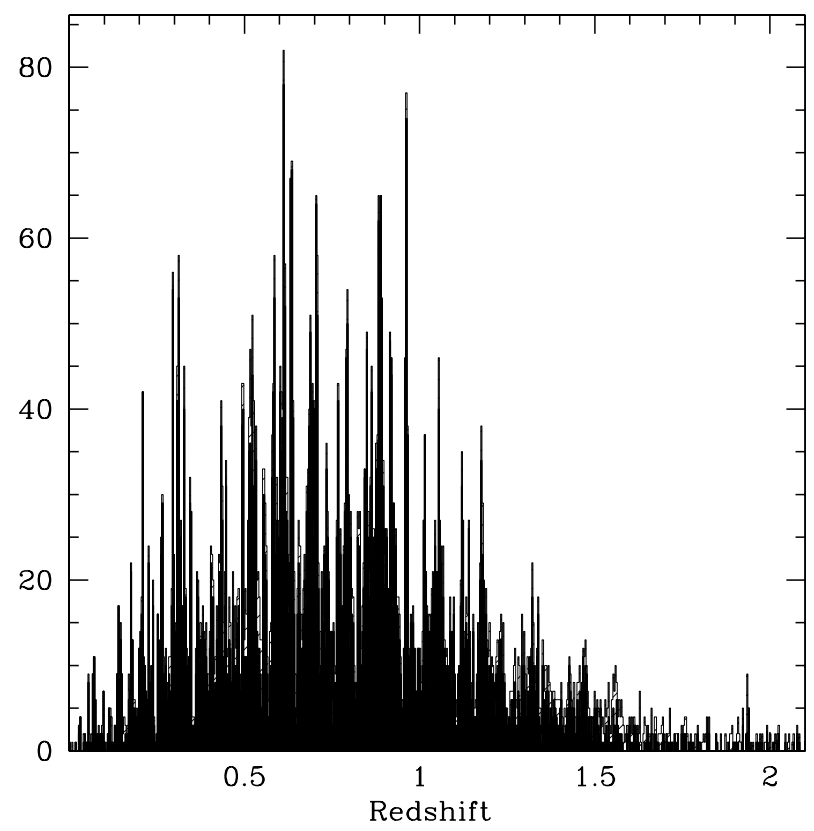

Fig. 26. Redshift distribution for galaxies in the VVDS-02h field with $0 \leq z \leq 1.7$, for galaxies with redshift quality flags $2,3,4,9$ (solid histogram) and galaxies with redshift quality flag 1 (dashed histogram). The redshift bin is $\mathrm{d} z=0.0033$.

Bondi, M., Ciliegi, P., Zamorani, G., et al. 2003, A\&A, 403, 857 Bottini, D., Garilli, B., Maccagni, D., et al. 2005, PASP, in press Boyle, B. J., Shanks, T., Croom, S. M., et al. 2000, MNRAS, 317, 1014

Brodwin, M., Lilly, S. J., Porciani, C., et al. ApJ [arXiv:astro-ph/0310038]

Cimatti, A., Daddi, E., Mignoli, M., et al. 2002, A\&A, 381, 68

Coleman, G. D., Wu, C. C., \& Weedman, D. W. 1980, ApJS, 43, 393

Colless, M. M., Dalton, G., Maddox, S., et al. 2001, MNRAS, 328, 1039

Conti, G., Mattaini, E., Maccagni, D., et al. 2001, PASP, 113, 452 
Giacconi, R., Zirm, A., Wang, J., et al. 2002, ApJS, 139, 369

Giavalisco, M., Dickinson, M., Ferguson, H. C., et al. 2004, ApJ, 600, L93

Horne, K. 1986, PASP, 98, 609

Ilbert, O., Tresse, L., Zucca, E., et al. 2005, A\&A, 439, 863

Iovino, A., McCraken, H. J., Garilli, B., et al. 2005, A\&A, accepted

Le Fèvre, O., Crampton, D., Lilly, S. J., Hammer, F., \& Tresse, L. 1995, ApJ, 455, 60

Le Fèvre, O., Hudon, D., Lilly, S. J., Crampton, D., Hammer, F., \& Tresse, L. 1996, ApJ, 461, 534

Le Fèvre, O., Vettolani, G., Maccagni, D., and the VVDS consortium, 2003a, The Messenger 111, 18

Le Fèvre, O., Vettolani, G., and the VVDS consortium, 2003b, International Astronomical Union Symp. 216, held 14-17 July, 2003 in Sydney, Australia, 216, 183

Le Fèvre, O., Mellier, Y., McCracken, H. J., et al. 2004a, A\&A, 417, 839

Le Fèvre, O., Vettolani, G., Paltani, S., et al. 2004b, A\&A, 428, 1043

Le Fèvre, O., Guzzo, G., Meneux, B., et al. 2005, A\&A, 439, 877

Lilly, S.J., Tresse, L., Hammer, F., Crampton, D., \& Le Fèvre, O. 1995a, ApJ, 455, 108

Lilly, S. J., Le Fèvre, O., Crampton, D., Hammer, F., \& Tresse, L. 1995b, ApJ, 455, 50
Lilly, S. J., Le Fèvre, O., Hammer, F., \& Crampton, D. 1996, ApJ, 460, L1

Lonsdale, C. J., Smith, H. E., Rowan-Robinson, M., et al. 2003, PASP, 115,897

McCracken, H. J., Radovich, M., Bertin, E., et al. 2003, A\&A, 410, 17

Madau, P., Pozzetti, L., \& Dickinson, M. 1998, ApJ, 498, 106

Marinoni, C., Le Fèvre, O., Meneux, B., et al., A\&A, submitted

Pierre, M., Valtchanov, I., \& Dos Santos, S. 2004, JCAP, 09, 011

Radovich, M., Arnaboldi, M., Ripepi, V., et al. 2004, 417, 51

Schade, D., Crampton, D., Hammer, F., Le Fèvre, O., Lilly, S. J. 1995, MNRAS, 278, 95

Schiminovich, D., Ilbert, O., Arnouts, S., et al. 2004, ApJ, 619, 47

Scodeggio, M., Franzetti, P., Garilli, B., et al. PASP, submitted

Shapley, A. E., Steidel, C. C., Pettini, M., \& Adelberger, K. L. 2003, ApJ, 588, 65

Steidel, C. C., Giavalisco, M., Pettini, M., Dickinson, M., \& Adelberger, K. L. 1996, ApJ, 462, L17

Steidel, C. C., Adelberger, K. L., Dickinson, M., et al. 1998, ApJ, 492, 428

Steidel, C. C., Shapley, A. E., Pettini, M., et al. 2004, ApJ, 604, 534

Vanzella, E., Cristiani, S., Dickinson, M., et al. 2005, A\&A, 434, 53

Wolf, C., Meisenheimer, K., Rix, H. W., et al. 2003, A\&A, 401, 73 